\title{
Experimental procedures for testing the performance of rail dampers
}

\author{
G. Squicciarini, M.G.R. Toward and D.J. Thompson \\ Institute of Sound and Vibration Research, University of Southampton, \\ Highfield, Southampton, SO17 1BJ, UK
}

Corresponding author address:

Dr. Giacomo Squicciarini

Institute of Sound and Vibration Research

University of Southampton

Southampton SO17 1BJ

UK

e-mail: g.squicciarini@ soton.ac.uk 


\section{Abstract}

Rail dampers work by increasing the attenuation with distance of vibration transmitted along the rail, a quantity known as the track decay rate. Currently, there are no standardized procedures to measure their effectiveness in reducing rolling noise without the need for in-track installation and time-consuming tests. This paper describes and evaluates experimental procedures for assessing rail dampers. Instead of field measurements it is proposed to use laboratory measurements of vertical and lateral decay rates on a free rail equipped with dampers. These are combined with in-situ measurements on an undamped track. The decay rates of a damped track can be approximated by adding the results of the damped free rail to those of the undamped track.

Three different methods are studied to measure the decay rates of damped free rails: (i) using a long rail, in the present work $32 \mathrm{~m}$ long, from frequency response functions measured at intervals along the rail; (ii) using a short rail, in the present work $6 \mathrm{~m}$ long, from the modal properties of the rail; and (iii) directly from the point and transfer frequency response functions at both ends of the short rail. The latter two are complementary: the modal method is more suited to low frequencies while the direct method is more suited to high frequencies. These methods are evaluated theoretically and by comparison with experimental results.

Good agreement is found between the various methods, for vibration in both vertical and lateral directions, between $300 \mathrm{~Hz}$ and $5 \mathrm{kHz}$. In practice, the direct short-rail method is likely to be sufficient for most applications. The limitations of the methods are identified and corrections are proposed for the effect of near-field waves in the rail.

Author Keywords: railway noise; damping; modelling; railway track; tuned absorber; rail damper; decay rates; rail pad

\section{Introduction}

In most situations, at least for conventional speeds, rolling noise originating from the interaction of the wheel and rail is the dominant source of environmental railway noise. It is caused by the combined surface roughness of the wheel and rail, which excites vibration of the wheel, rail and sleepers. In turn this vibration radiates sound. Models for this phenomenon have been developed and validated against field measurements [14]. Generally, the sleeper is found to be the major source of noise below around $400 \mathrm{~Hz}$, the rail is dominant between 400 and $1600 \mathrm{~Hz}$ and the wheel is of increasing importance 
at higher frequencies [5]. Both the track and the wheels can contribute significantly to the overall noise level. Their relative contributions depend on the roughness spectrum, the train speed and various design parameters of the wheel and track. Particularly important is the track decay rate, the rate of attenuation of vibration along the rail, which governs the sound radiated by the rail $[6,7]$. In order to separate the wheel and track components, either advanced experimental techniques [8] or theoretical models [1, 2] are required.

Theoretical models are also important to facilitate the development of appropriate noisemitigating solutions. Increasing the stiffness (and damping) of the rail pads, fitted between the rail and sleepers, can decrease the rail component of rolling noise by increasing the track decay rate and hence reducing the effective radiating length of rail. Conversely this can increase the noise radiated from the sleeper due to the increased coupling [9]. Among the various alternatives developed in the last decades [10] this paper focuses on rail dampers, which are a good and efficient candidate for reducing the noise at source [11]. Rail dampers are particularly effective when tracks are fitted with soft pads (low strain stiffness below $250 \mathrm{MN} / \mathrm{m}$ ).

Various forms of rail damper have been designed to increase the track decay rate without the need for higher pad stiffness [12-15]. Usually, such dampers are bolted or clipped onto the rail between the sleepers. They are designed to be effective primarily for the vertical vibration of the rail as, for a wheel rolling on a straight track, this is the most important direction of excitation. However, as the vertical and lateral directions are coupled, and the lateral decay rates are often lower than the vertical ones, the lateral vibration can also contribute to the noise and this has to be accounted for in a numerical model for noise radiation. Overall reductions in noise level ranging from 2 to $6 \mathrm{~dB}(\mathrm{~A})$ have been obtained in comparative measurements of various types of damper installed on a track [15-17]. Reductions are greatest for a track with softer rail pads where the rail component of rolling noise is more dominant due to the low initial decay rates [17], whereas noise reduction on tracks equipped with stiff pads can be as low as $1 \mathrm{~dB}(\mathrm{~A})$. Currently, there is no standardized method to assess the in-situ performance of dampers. Such an assessment would require field tests following their installation in a track, which can be expensive to carry out. An international project, STARDAMP [18], was therefore set up with the aim of developing standard procedures to verify wheel and rail damper effectiveness without the need for full installation in the field. 
The aim of this paper is to present the theoretical basis behind the method developed to predict the acoustic performance of rail dampers and to evaluate it in order to establish its range of applicability. This method consists of a combined experimental-numerical procedure and can be summarised in the following steps. First, measurements are made of the decay rates of a length of free rail fitted with rail dampers. Second, those of the undamped target track are measured. To estimate the decay rates of the damped track, the two results are added. Finally, the track decay rates of the damped and undamped cases are used in a prediction model to determine the acoustic effect of the dampers. This is expressed in terms of the reduction in acoustic power radiated by the track or the overall noise reduction for a given wheel design. The advantages of this approach are that it avoids expensive field tests and that the results can be used for different target situations, including different track designs, based on the same laboratory test results. A requirement of the proposed method is an accurate and repeatable procedure for determining the decay rates of a free rail fitted with dampers. These have previously been obtained from measurements of a $4 \mathrm{~m}$ section of freely supported rail [15]. These were determined at low frequency from the modal properties of the rail, and at high frequency directly from point and transfer frequency response functions (FRFs) at either end of the rail. The decay rates of a damped free rail can also be derived from FRFs measured at intervals along a longer section of rail, similar to the track decay rate method described in EN15461:2008 [6]. However, this approach has not previously been tested for free rails. In this paper, methods based on the three experimental approaches outlined above are tested, compared and critically evaluated.

The expected reduction in acoustic power from the track in each one-third octave band, $\Delta L$, can be calculated from the undamped track decay rate, $D R_{\mathrm{u}}$ and the damped track decay rate, $D R_{\mathrm{d}}$, according to [5, 7]:

$\Delta L=10 \log _{10} \frac{D R_{u}}{D R_{d}}$

However, the main assumptions behind eq. (1) are that there is only one wave propagating in the rail and that three-dimensional effects on the rail radiation ratio can be neglected (see [19]). The in-situ performance of dampers will depend not only on their effect on the rail noise but also on the relative contributions of the wheels and individual track components to the overall noise. These contributions can be predicted using models such as TWINS [1]. A software tool that implements TWINS-like predictions of rolling noise has been developed by the authors within the STARDAMP project [20, 21]. 


\section{Measurements of track decay rate: in situ, test track and 'long rail' methods}

\section{$2.1 \quad$ Method based on EN 15461}

Track decay rates are measured in the field using a method described in EN 15461:2008 [6]. This relies on measurements of the driving-point frequency response function (FRF) at a reference point $A\left(x_{0}\right)$, and the transfer FRFs, $A\left(x_{n}\right)$, between the reference position and the other points on a measurement grid defined by a variable spacing $\Delta x_{n}$. The decay rate is given by

$$
D R=\frac{4.343}{\sum_{n=0}^{n_{\max }} \frac{\left|A\left(x_{n}\right)\right|^{2}}{\left|A\left(x_{0}\right)\right|^{2}} \Delta x_{n}}
$$

The derivation of this equation was first presented in [22] and proposed again in [7] where the advantages were shown of a procedure that considers the summed response of the rail instead of fitting a decay curve assuming the presence of one wave type only. Eq.

(2) in fact approximates the analytical calculation of the overall decay as:

$$
\frac{1}{2 \beta}=\int_{0}^{\infty} \frac{|A(x)|^{2}}{|A(0)|^{2}} \mathrm{~d} x \approx \sum_{x_{i}=0}^{x_{\max }} \frac{\left|A\left(x_{i}\right)\right|^{2}}{|A(0)|^{2}} \Delta x_{i}
$$

where $\beta$ is an overall decay constant accounting for all the waves travelling into the rail and is related to decay rate by $D R=20 \log _{10} \mathrm{e}^{\beta}$. This method was adopted as a standard for track decay rates measurements [6]. Although this standard specifies the spacing $\Delta x_{n}$ this has been redefined in the present application to allow for the higher decays due to added dampers. The measurement grid adopted in the present work is described and discussed further below.

\subsection{Test track measurements}

Measurements have been carried out on a test track located at the University of Southampton which was designed to be broadly representative of modern circulated track within the UK. It has a length of $32 \mathrm{~m}$ and is fitted with UIC 60 rail, 51 concrete monobloc sleepers with a mean spacing of $0.63 \mathrm{~m}$ (standard deviation $0.03 \mathrm{~m}$ ), $10 \mathrm{~mm}$ studded natural rubber pads with an effective vertical stiffness of approximately 120 
$\mathrm{MN} / \mathrm{m}$ and compacted granite ballast to a depth of $0.3 \mathrm{~m}$. Measurements were conducted without dampers and with two dampers referred to as $\mathrm{A}$ and $\mathrm{B}$.

A measurement grid was marked up from a reference point located at the centre of the $10^{\text {th }}$ sleeper span, corresponding to $5.96 \mathrm{~m}$ from the rail end. Measurements were made at $1 / 4$-sleeper intervals from this point up to the $16^{\text {th }}$ sleeper span, then at the following mid-span positions: $17^{\text {th }}, 18^{\text {th }}, 20^{\text {th }}, 22^{\text {nd }}, 26^{\text {th }}, 30^{\text {th }}, 34^{\text {th }}, 38^{\text {th }}, 42^{\text {nd }}$ and $46^{\text {th }}$, resulting in a total of 35 points covering $23 \mathrm{~m}$ of the rail. This measurement grid differs from the one proposed in [6], containing more points close to the excitation point and being shorter. Dampers added to the rail cause waves to decay at a higher rate, requiring a higher resolution close to the excitation point.

A miniature accelerometer was attached to the rail at the reference point. For vertical measurements this was on top of the rail at the centre of the rail head, while for lateral measurements it was on the side of the rail head. The rail was excited with an instrumented hammer. Mobility FRFs (i.e. velocity over force) were used throughout the analysis with a frequency resolution of $3 \mathrm{~Hz}$. Decay rates in each one-third octave band up to $5 \mathrm{kHz}$ were calculated using eq. (2). To obtain one-third octave band decay rates the modulus-squared of FRFs $\left(A\left(x_{0}\right)\right.$ and $\left.A\left(x_{n}\right)\right)$ are averaged into one-third octave bands before applying eq. (2).

As track decay rates have been found to be dependent on temperature, rail temperatures were measured at the start and end of each measurement set using a thermocouple attached to the underside of the rail; average temperatures are reported.

The procedure adopted was originally designed to be applied on an infinitely long track [6]. In the standard it is recommended to measure over 66 sleeper bays so as to estimate correctly also the lowest values of decay. By assuming a minimum acceptable difference between the response level at the driving point and the last point measured of at least 10 $\mathrm{dB}$ (as in [6]) it is found that, in the present application, the lowest detectable decay rate is at about $0.5 \mathrm{~dB} / \mathrm{m}$.

The finiteness of the test track can be observed in the measured FRFs when shown in narrow band form: they show additional peaks (and dips) corresponding to the vibration modes of the finite length rail. If results are shown in one-third octave bands, this frequency averaging smoothens the measured FRFs so that the reflections from the ends are no longer visible. As an example of this behaviour Figure 1(a) presents the vertical driving point mobility as measured on the test track in the undamped configuration, 
both in narrow band and in one-third octave band form. Up to $500 \mathrm{~Hz}$ the effect of finiteness of the test track only causes small fluctuations. Above $500 \mathrm{~Hz}$ the waves start to propagate in the rail and vibration modes are clearly visible in the narrow band mobility. For this test track there are 2 or 3 modes in each one-third octave band. The one-third octave band mobility shows the most important features found on a real track even above $500 \mathrm{~Hz}$ : a sharper peak at around $1 \mathrm{kHz}$ representing the 'pinned-pinned' mode and another sharp peak at $5 \mathrm{kHz}$ due to a second wave type (foot flapping [23]) cutting on in the rail.

Figure 1(b) shows the driving point mobility in the lateral direction measured at the rail head. Fluctuations due to vibration modes in the narrow-band FRF are larger than in the vertical direction due to a lower decay rate. The modal density is also higher as, along with lateral bending, torsion is also excited [24, 25]. A one-third octave band representation again shows the most important features such as bouncing of the rail above ballast and pad lateral stiffness below $300 \mathrm{~Hz}$, 'pinned-pinned' resonance at 500 $\mathrm{Hz}$ and the correct level at higher frequencies.

These limitations related to the relatively short length of the track are of importance only in the case of the undamped track; the addition of dampers greatly increases the minimum decay rate in the track, as will be seen in the results below.

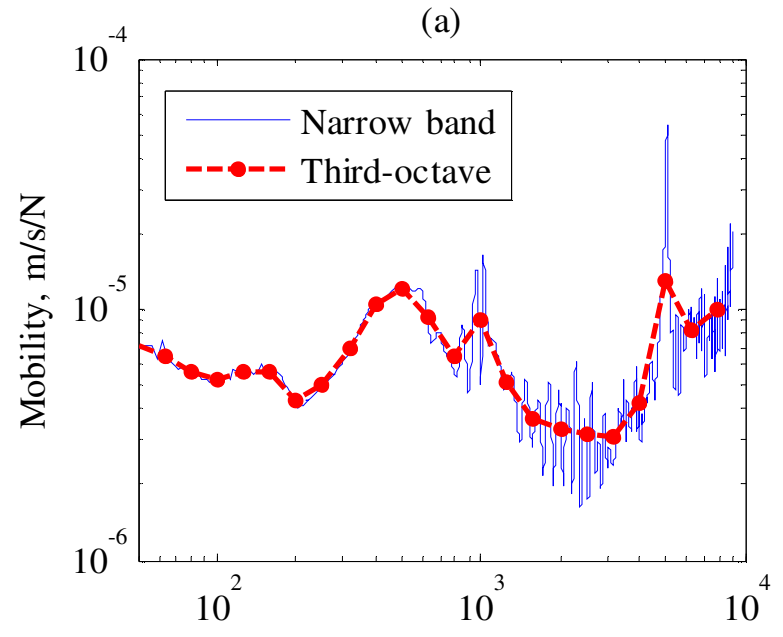

Frequency, $\mathrm{Hz}$

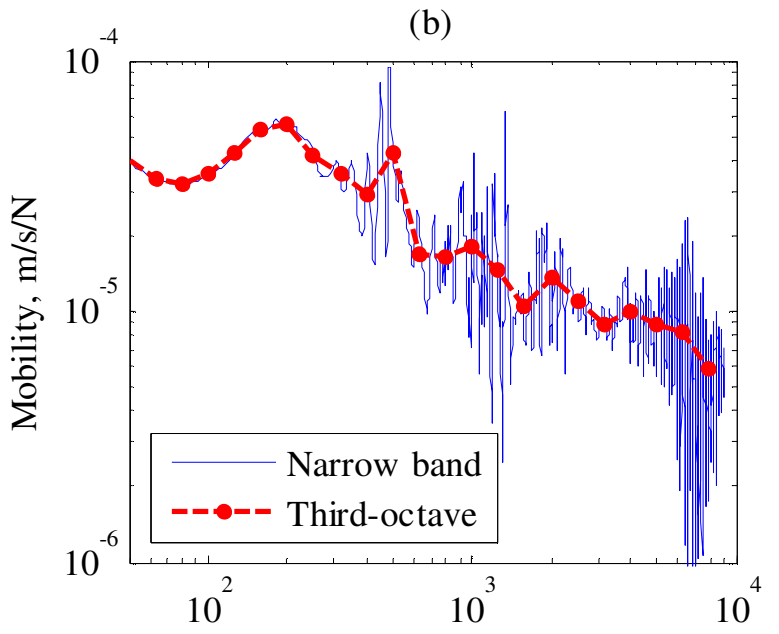

Frequency, $\mathrm{Hz}$

Figure 1. Vertical (a) and lateral (b) driving point mobility measured on the $32 \mathrm{~m}$ long test track.

Measured track decay rates are shown in Figure 2 for this test track without dampers and when fitted with the two different rail dampers. Note that a logarithmic scale is used to plot decay rates as a consequence of the relation given in eq. (1). Measured decay rates of vertical rail vibration are shown in Figure 2(a). For the undamped track, there is high attenuation at low frequencies because of the blocking effect of the stiffness of the 
foundation [5]. Above around $500 \mathrm{~Hz}$ the decay rate decreases to a minimum at $2 \mathrm{kHz}$, before increasing again to a peak at around $5 \mathrm{kHz}$. Decay rates measured on the damped track show a significant increase between 500 and $4000 \mathrm{~Hz}$.

The measurement results for the lateral direction are shown in Figure 2(b). These show similar trends, although the lateral decay rates of the undamped track are lower than the corresponding vertical ones at most frequencies, dropping at a lower frequency due to the lower pad stiffness for this direction. The undamped track has a peak at $3150 \mathrm{~Hz}$ due to the effect of higher order wave modes [24]. Note that in both directions the decay rate has a minimum value of about $0.5 \mathrm{~dB} / \mathrm{m}$ which can be affected by the finite length of the track; this will be considered further in Section 3.

(a)

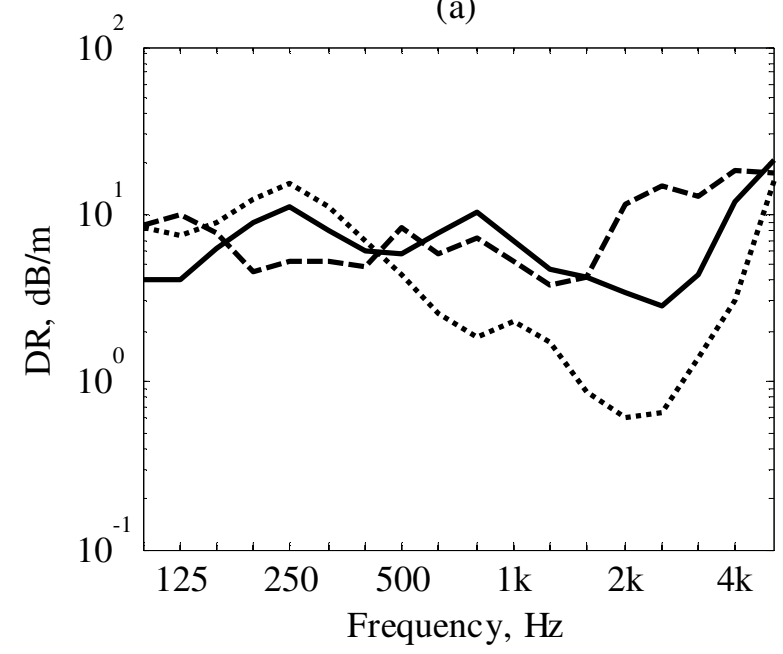

(b)

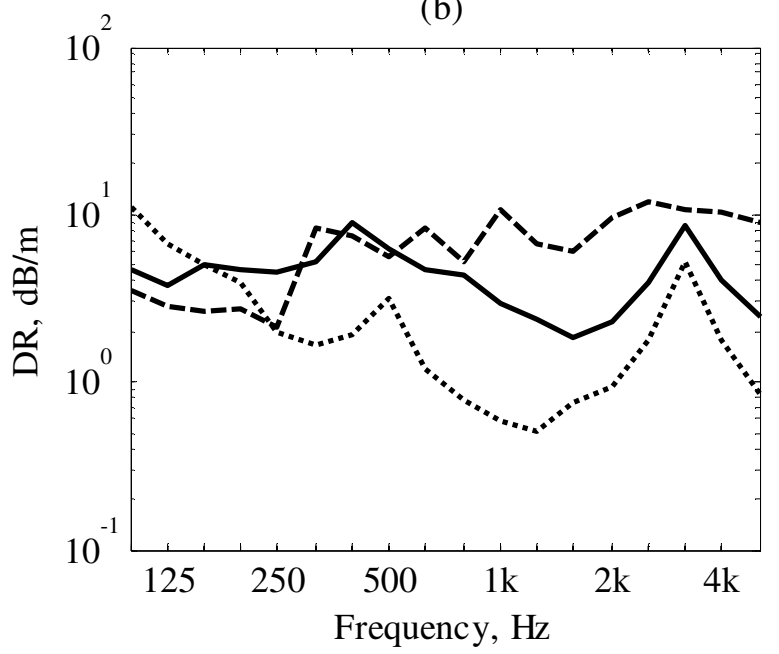

Figure 2: Decay rates measured on the test track in vertical (a) and lateral (b) direction.

(a) … Undamped track $\left(11^{\circ} \mathrm{C}\right) ;$ - damper A $\left(11^{\circ} \mathrm{C}\right) ;---\operatorname{damper} \mathrm{B}\left(15^{\circ} \mathrm{C}\right)$

(b) …. Undamped track $\left(6.5^{\circ} \mathrm{C}\right)$; - damper A $\left(10^{\circ} \mathrm{C}\right) ;---\operatorname{damper} \mathrm{B}\left(14^{\circ} \mathrm{C}\right)$

\subsection{Long free-rail method for finding decay rates associated with dampers}

For a damped free rail that is long enough, vertical and lateral decay rates can be measured using a method based on that described in [6], i.e. using eq. (1).

To illustrate this, measurements were conducted on a $32 \mathrm{~m}$ long free rail of the test track described above. One of the rails was unclipped from the track and supported on soft springs formed of sections of hydraulic hose, resulting in a bounce mode of the rail at approximately $15 \mathrm{~Hz}$. The same measurement points as described in Section 2.2 have been adopted.

Results from this method are shown in Figure 3. The trend is similar in both vertical and lateral directions and for both damper types. The decay rate is low below $500 \mathrm{~Hz}$; in this 
range the decay rate of the track is already high and dampers are not designed to be effective. Above $500 \mathrm{~Hz}$ the dampers become effective and the decay rate of the damped free rail is higher than the decay rate of the undamped track (Figure 2). Results of Figure 3 will be shown again in Section 5 for comparison with those of the short rail methods which are considered in Section 4.

(a)

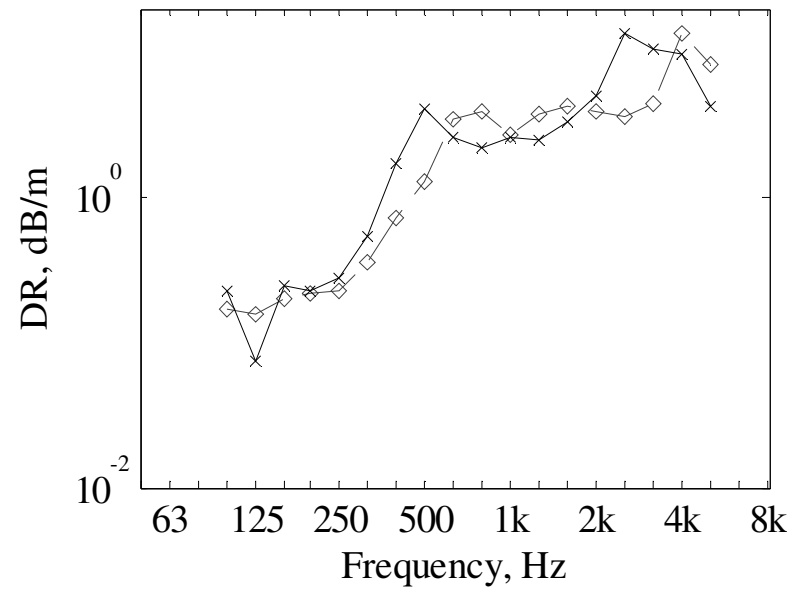

(b)

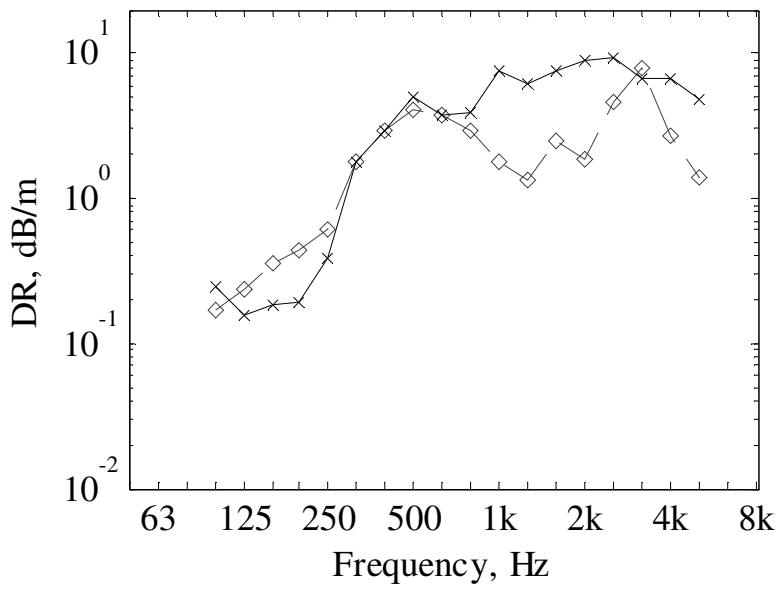

Figure 3. Decay rate of free rail in vertical (a) and lateral (b) direction.

(a) $-\diamond-$, damper $\mathrm{A}\left(15^{\circ} \mathrm{C}\right) ;-\times-$, damper $\mathrm{B}\left(11^{\circ} \mathrm{C}\right)$.

(b) $-\diamond-$, damper $\mathrm{A}\left(10^{\circ} \mathrm{C}\right) ;-\times-$, damper $\mathrm{B}\left(14.2^{\circ} \mathrm{C}\right)$.

\section{Effect of evanescent waves and finite length track on decay rate estimates}

In this section, analytical models are introduced to explore the limitations of the measurement methods introduced in Section 2. The method described in Section 2.1 (and [6]) determines the total decay rate of the track, which includes the effect of evanescent waves in the vicinity of the driving point. Therefore the decay rate obtained using eq. (2) tends to be higher than the equivalent decay of the propagating wave (see [7]). Similarly, applying the same method on a test track of finite length (Section 2.2) would again include the effect of evanescent waves close to the forcing point and would moreover be affected by waves reflected at the ends. Again, decay rates measured according to the standard method would differ from those of the propagating wave only. The long rail method described in Section 2.3 would also encounter similar features, being performed on a finite length rail.

In predicting rolling noise with numerical models, the measured track decay rates accounting for all the waves are often used as input data to represent the decay rate of the propagating wave only, assuming that the contribution of other waves to the overall response is negligible. This section aims to quantify the effect of this assumption using 
models of a rail based on a beam. In particular, with the aim of improving the understanding on the measurements described in Section 2, four different cases are considered: (i) an infinite unsupported beam, which provides a useful introductory example for the following cases. (ii) A finite unsupported beam, which is representative of the long rail method (described in Section 2.3). (iii) An infinite beam on a double layer elastic foundation, which is representative of a real track. (iv) A finite length beam on a double elastic foundation, which represents a test track of finite length, as the one used in this work (see Section 2.2). The ratio between the decay rate calculated considering the contribution of all the waves (using eq. (2) with FRFs estimated from models) and the decay rate obtained directly from the model corresponding to the propagating wave only (imaginary part of the wavenumber) is presented for each case and represented as $D R_{t o t} / D R_{p}$.

Regardless the way the FRFs used in eq. (2) are obtained (analytical models or measurements), the maximum length and the spacing of the calculation grid limit the range of validity of measured decay rates. In the case of numerical calculations the grid can be arbitrarily defined to overcome this limitation. However, for those cases representing an arrangement of the rail/track corresponding to a real measurement situation, it is important to maintain the same grid. In this way the ratio $D R_{t o t} / D R_{p}$ is equally affected by the grid in the measurements and in the model. For the first case presented (infinite unsupported beam) the measurement grid adopted is refined and extended in such a way that the grid itself does not affect the results in the range considered. Measurements on a real track have not been considered in this paper; therefore, to simplify the interpretation of the result, the grid for this case has also been refined and extended so that it does not affect the outcomes. The case of a finite length rail is representative of the long rail method and so the measurement grid used is the same as adopted in the measurements. For a finite length beam on an elastic foundation (representing a test track), the grid adopted in the numerical calculation is the same as in the measurements.

The Timoshenko beam models are representative of the vertical bending behaviour of the rail up to at least $2 \mathrm{kHz}$ [7]; the Euler-Bernoulli beam model is also introduced as its simplicity allows additional insight to be gained. For the lateral vibration of a rail more complex models would be more appropriate, e.g. [24, 25], but the main trends are still expected to be similar. All the models adopted rely on a continuous support below the rail, in practice the rail is supported by discrete sleepers resulting in pinned-pinned 
resonance, in the point mobility, and in a blocked region in the decay rate. The effect these have on pass-by noise is believed to be small [5] and discrete support is not modelled in this paper,

\subsection{Decay rates of an infinite unsupported beam}

Consider an infinite beam excited by a harmonic force of amplitude $F$ at $x=0$. The equations of motion for a Timoshenko beam are [26]:

$G A \kappa \frac{\partial}{\partial x}\left(\phi-\frac{\partial u}{\partial x}\right)+\rho A \frac{\partial^{2} u}{\partial t^{2}}=F \delta(x) e^{i \omega t}$

$G A \kappa\left(\phi-\frac{\partial u}{\partial x}\right)-E I \frac{\partial^{2} \phi}{\partial x^{2}}+\rho I \frac{\partial^{2} \phi}{\partial t^{2}}=0$

The two variables $u$ and $\phi$ represent the transverse displacement and the rotation of the section, respectively. The product of the shear modulus $G$, the area $A$ and the shear coefficient $\kappa$ represents the shear stiffness $(G A \kappa), E$ is the Young's modulus, $I$ the second moment of area ( $E I$ the bending stiffness), $\rho$ is the density ( $\rho I$ the rotational inertia). Damping is introduced by making the Young's modulus (and also the shear modulus) complex by means of the loss factor $\eta$ as $E=E_{0}(1+i \eta)$.

Considering free vibration and assuming a harmonic solution at circular frequency $\omega$, $u=A_{n} e^{-i k x} e^{i \omega x}$, equations (4) and (5) can be rearranged into a single equation and the following characteristic equation can be found:

$k^{4}+\left(\frac{m \omega^{2}}{G A \kappa}-\frac{\rho I \omega^{2}}{E I}\right) k^{2}+\frac{\rho A \omega^{2}}{E I}\left(1-\frac{\rho I \omega^{2}}{G A \kappa}\right)=0$

The solution of this yields four wavenumbers $k$ at each frequency: two for the waves propagating or decaying in the positive direction and two for the waves propagating or decaying in the negative direction. In the absence of damping, one of the two pairs of waves has a wavenumber which is always real, representing a propagating wave, and this is written as $\pm k_{p}$. The other pair of waves has a wavenumber that is purely imaginary at low frequency. This will be referred to as an evanescent wave and its wavenumber represented as $\pm k_{e}$. However, there exists a frequency at which this wave 'cuts on', i.e. its wavenumber becomes real. The same nomenclature is adopted when 
damping is introduced in the beam although then both waves have complex wavenumbers.

The decay rate can be expressed in $\mathrm{dB} / \mathrm{m}$ and, for the propagating wave, is written as (see [5])

$$
D R_{p}=-8.686 \operatorname{Im}\left(k_{p}\right)
$$

To find the overall decay rate it is necessary to solve for the forced response. The solutions in the positive and negative domain are:

$$
\begin{array}{ll}
u^{+}(x)=A_{2} e^{-i k_{e} x}+A_{4} e^{-i k_{p} x} & \text { for } x \geq 0 \\
u^{-}(x)=A_{1} e^{i k_{e} x}+A_{3} e^{i k_{p} x} & \text { for } x \leq 0
\end{array}
$$

The coefficients $A_{n}$ are obtained by applying the appropriate boundary conditions at $x=0$ and solving a system of equations for the four unknown wave amplitudes. Similarly, for an Euler-Bernoulli beam the response is given by equation (8) but now the wavenumbers are given by

$k_{p}=\left(\frac{\rho A \omega^{2}}{E I}\right)^{1 / 4} ; k_{e}=-i\left(\frac{\rho A \omega^{2}}{E I}\right)^{1 / 4}$

while the wave amplitudes are equal to

$$
A_{1}=A_{3}=-i A_{2}=-i A_{4}=-F / 4 E I k_{p}^{3}
$$

In order to determine the overall decay rate, the response given by equation (8) for a unit force, $F=1$, is evaluated at several points along the beam. The relative amplitude is plotted in Figure 4 against distance, normalised by wavelength $\lambda=2 \pi / \operatorname{Re}\left(k_{p}\right)$, for an Euler-Bernoulli beam. Parameters have been chosen to be representative of the vertical bending of a UIC60 rail (see Appendix A, Table A 1). The damping loss factor is assigned values ranging from 0.02 to 0.8 . It can be seen that far from the excitation point the decay is exponential (linear in terms of decibels) but the evanescent wave increases the response in the vicinity of the excitation point by $3 \mathrm{~dB}$. The corresponding results for the Timoshenko beam are similar although they depend on frequency. 


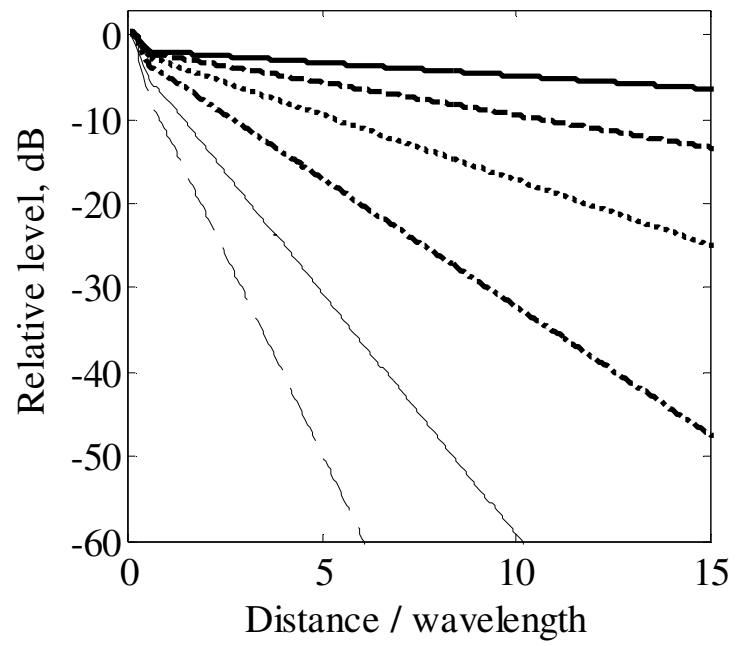

Figure 4: Vibration level plotted against distance for unsupported Euler-Bernoulli beam with various damping loss factors. Thick lines:,$- \eta=0.02 ;---, \eta=0.05 ; \cdots \cdots, \eta=0.1 ;-\cdot-\cdot, \eta=0.2$; thin lines:,$- \eta=0.4 ;---, \eta=0.8$.

Results obtained from eq. (8) are then used as the FRFs in eq. (2). The resulting decay rate $\left(D R_{\text {tot }}\right)$ can be compared with the decay of the propagating wave only $\left(D R_{p}\right)$ from eq. (7). The results are shown in Figure 5 as the ratio $D R_{t o t} / D R_{p}$.

For the Euler-Bernoulli beam, the results are found to be independent of frequency. The ratio $D R_{\text {tot }} / D R_{p}$ is plotted against loss factor in Figure 5(a). It can be seen that the ratio tends to 2 for low damping and reduces as the loss factor increases. The factor of 2 corresponds to the ratio of the squared amplitude of the total response at $x=0$ and the response of the propagating wave component (see eq. (10)).

For a Timoshenko beam the corresponding results are dependent on frequency as shown in Figure 5(b). At low frequency the results tend to those of the corresponding EulerBernoulli beam, while above $1 \mathrm{kHz}$ they tend to unity as the amplitude of the evanescent wave reduces. 
(a)

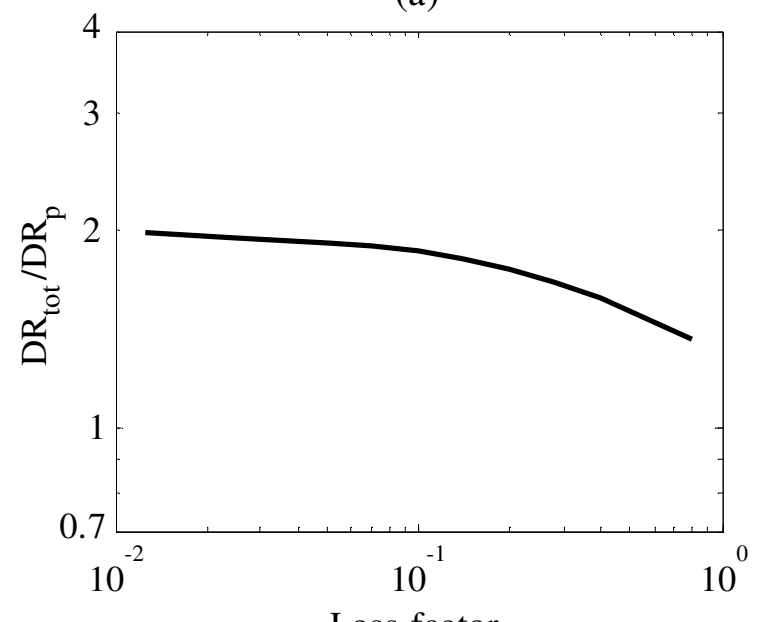

(b)

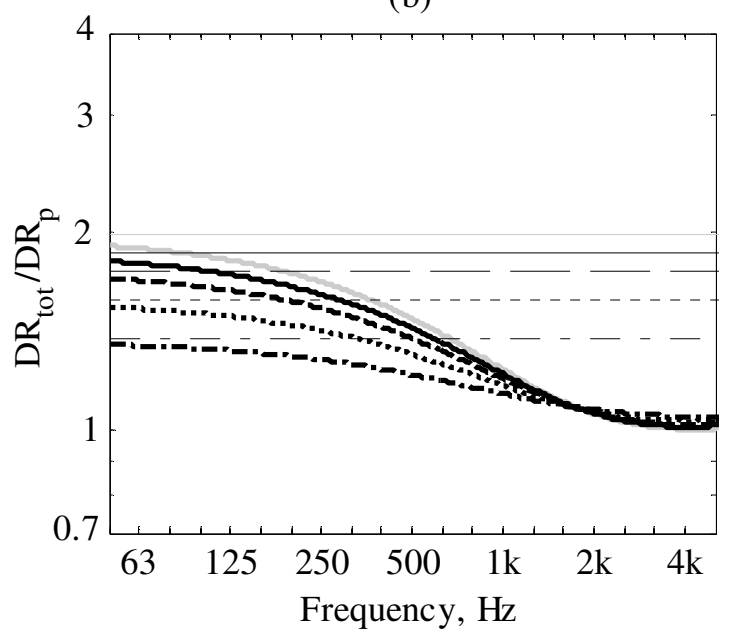

Figure 5: Ratio of total decay rate to decay rate of propagating wave for various loss factors. (a)

Unsupported Euler-Bernoulli beam. (b) Thin lines: unsupported Euler-Bernoulli beam. Thick lines: unsupported Timoshenko beam. - , $\eta=0.02 ;-, \eta=0.1 ;---, \eta=0.2 ; \cdots \cdots, \eta=0.4 ;-\cdot-\cdot$, $\eta=0.8$.

\subsection{Decay rates of an unsupported finite length beam}

In the case of a finite length beam, forced at distance $L_{1}$ from one end and $L_{2}$ from the other, the forced response can be found from the four waves in equation (8) and four more introduced by reflections from the ends. This model can be considered as a representation of the long rail method of Section 2.3.

Eq. (6) is still valid for calculating the wavenumbers in this situation while the solutions in the positive and negative $x$ domain can now be written as:

$$
\begin{array}{ll}
u^{+}(x)=A_{5} e^{i k_{p} x}+A_{6} e^{-i k_{p} x}+A_{7} e^{i k_{e} x}+A_{8} e^{-i k_{e} x} & \text { for } x \geq 0 \\
u^{-}(x)=A_{1} e^{i k_{p} x}+A_{2} e^{-i k_{p} x}+A_{3} e^{i k_{e} x}+A_{4} e^{-i k_{e} x} & \text { for } x \leq 0
\end{array}
$$

The wave amplitudes are found by applying continuity at the forcing point as before as well as free boundary conditions at the ends. The ratio between the total decay rate, obtained by applying eq. (2), and the decay of the propagating wave only is shown in Figure 6 for four different loss factor values. Both narrow band and one-third octave band results are presented where the latter are determined from one-third octave band FRFs. The lowest loss factor (0.02), normally used for the undamped rail [5], is excluded from the analysis as this case is meant to represent a damped rail only. The total length of the beam and the location of the forcing point have been set according to the measurements made for the long rail method (Section 2), with $L_{1}=6 \mathrm{~m}$ and $L_{1}+L_{2}=32 \mathrm{~m}$. 
The overall trend is similar to the results of the infinite beam but the reflections at the end generate a series of sharp peaks, the amplitude of which depends on the damping.

(a)

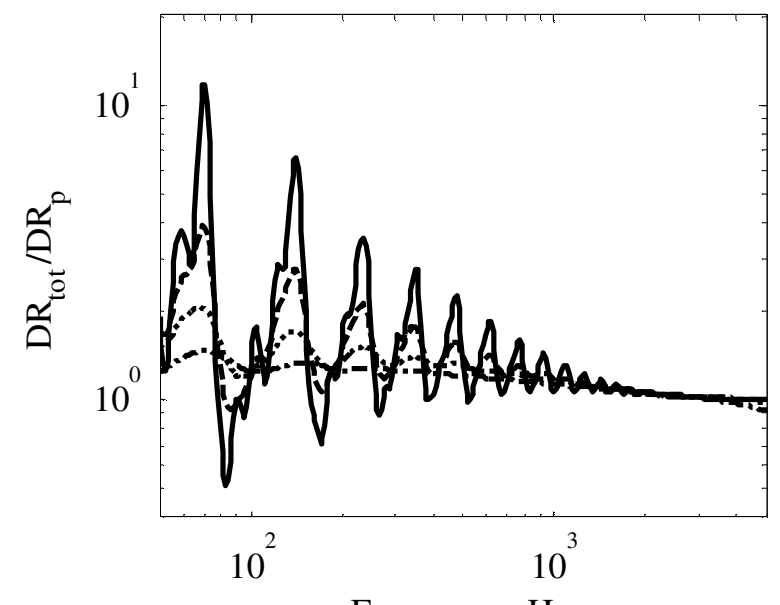

(b)

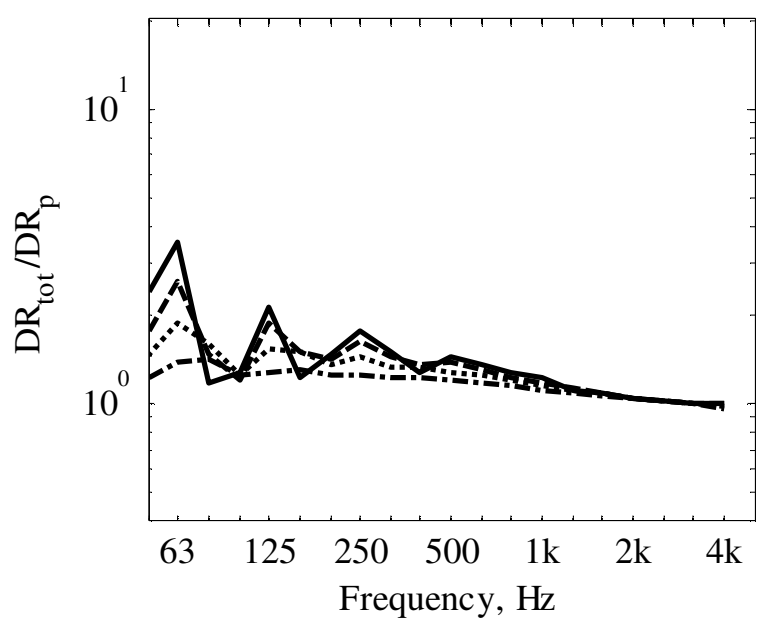

Figure 6: Ratio of total decay rate to decay rate of propagating wave of a finite length $(32 \mathrm{~m})$ unsupported Timoshenko beam for various damping loss factors. -,$\eta=0.1 ;---, \eta=0.2 ; \ldots \ldots$, $\eta=0.4 ;-\cdot-\cdot, \eta=0.8$. (a) Narrow-band; (b) one-third octave band.

\subsection{Decay rates of an infinite beam on two-layer foundation}

The case of an infinite beam supported by a double elastic foundation, as shown in Figure 7 , is often used to study wave propagation in a railway track where the two layers of damped springs represent pad and ballast stiffness while the intermediate masses represent the sleepers.

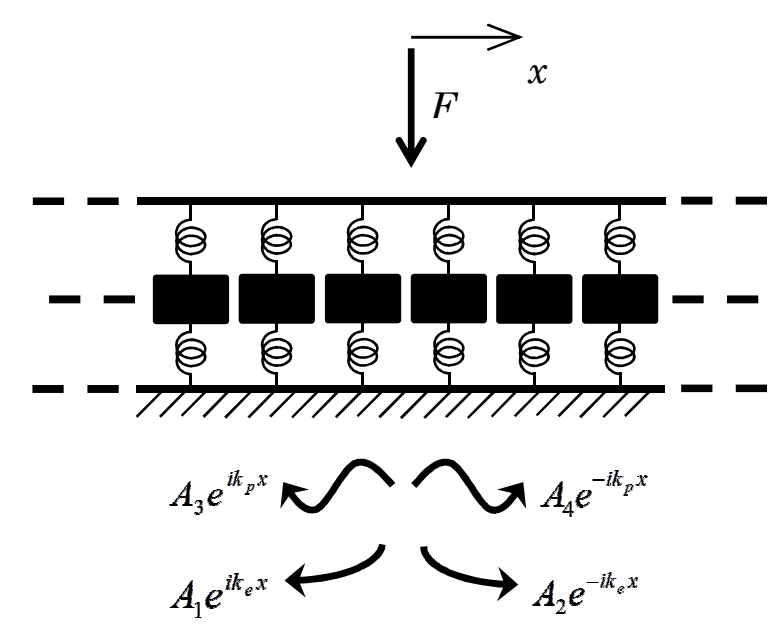

Figure 7: Schematic representation of waves propagating in an infinite beam on a two-layer support forced at $x=0$.

Equation (4) can be modified to account for the elastic foundation as [5]: 
$G A \kappa \frac{\partial}{\partial x}\left(\phi-\frac{\partial u}{\partial x}\right)+s u+\rho A \frac{\partial^{2} u}{\partial t^{2}}=F \delta(x) e^{i \omega t}$

where $s$ is a frequency dependent support stiffness per unit length given by:

$s=\frac{s_{p}\left(s_{b}-\omega^{2} m_{s}\right)}{s_{p}+s_{b}-\omega^{2} m_{s}}$

$s_{p}$ and $s_{b}$ are the pad and ballast dynamic stiffnesses per unit length for one rail, respectively. They are assumed to be constant and are made complex with loss factors $\eta_{p}$ and $\eta_{b}$ respectively. $m_{s}$ is the sleeper mass per unit length. Eq. (5) is not modified by the presence of the support. Instead, the support stiffness modifies the characteristic equation (eq. (6)) which is now:

$k^{4}+\left(\frac{s-\rho A \omega^{2}}{G A \kappa}-\frac{\rho I \omega^{2}}{E I}\right) k^{2}+\frac{s-\rho A \omega^{2}}{E I}\left(1-\frac{\rho I \omega^{2}}{G A \kappa}\right)=0$

The solution is found as in eq. (8), having updated the wavenumber from eq. (14).

Figure 8 shows the decay rate as calculated from this model including propagating and evanescent waves (Figure 8(a)) and in terms of the propagating wave only (Figure 8(b)). For this case the rail loss factor typical of the undamped track (0.02) is also included to represent the measurement on the undamped test track described in Section 2 . In addition, a comparison between this figure and the measurements of Figure 2 suggests that a rail loss factor between 0.2 and 0.8 would be necessary in this model to simulate the effect of dampers. The other track parameters adopted are listed in Appendix B. 
(a)

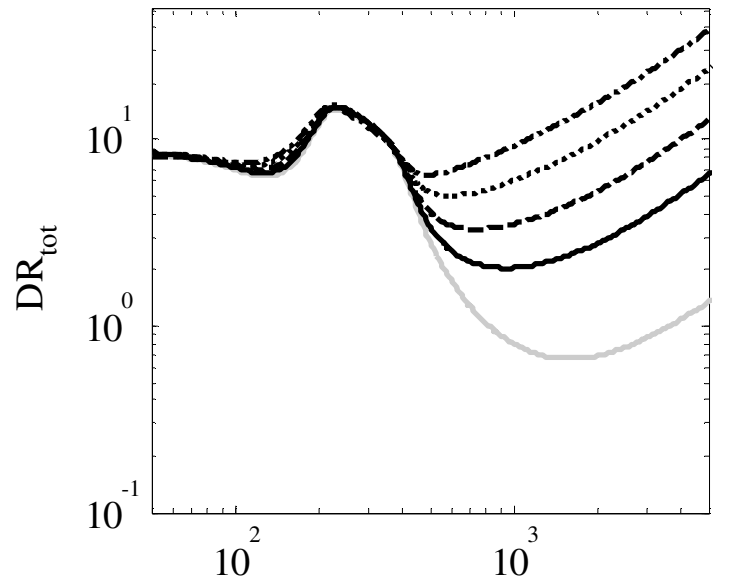

Frequency, $\mathrm{Hz}$ (b)

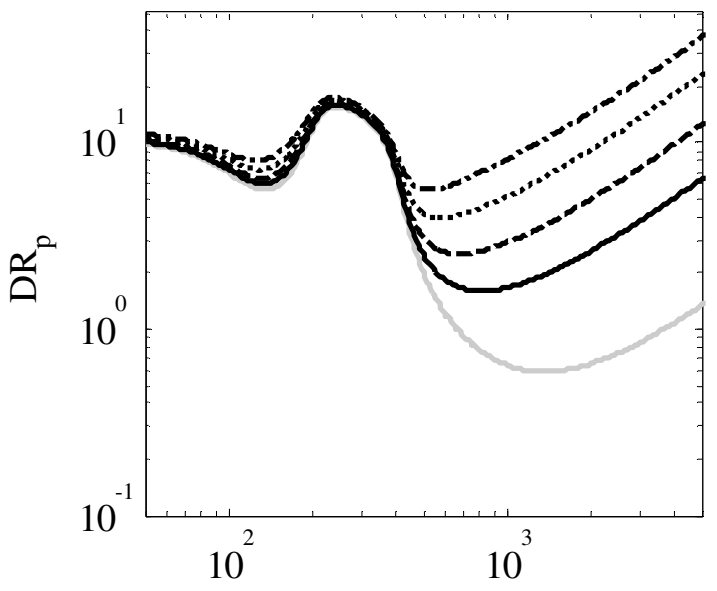

Frequency, $\mathrm{Hz}$

Figure 8: Decay rate of an infinite Timoshenko beam on a two layer foundation for various damping loss factors of the rail.,$- \eta=0.02 ;-, \eta=0.1 ;---, \eta=0.2 ; \cdots \ldots, \eta=0.4 ;-\cdot-\cdot, \eta=0.8$.

(a) Total decay rate; (b) decay rate of propagating wave only.

Figure 9 shows the ratio of the total decay rate to that of the propagating wave.

Compared with Figure 6, the effect of the support stiffness modifies this result below 500 $\mathrm{Hz}$, where it oscillates between 0.75 and 1.4. Above this frequency the waves tend to propagate freely along the rail and the ratio tends to the free rail result. There is little difference between narrow-band and one-third octave results.

(a)

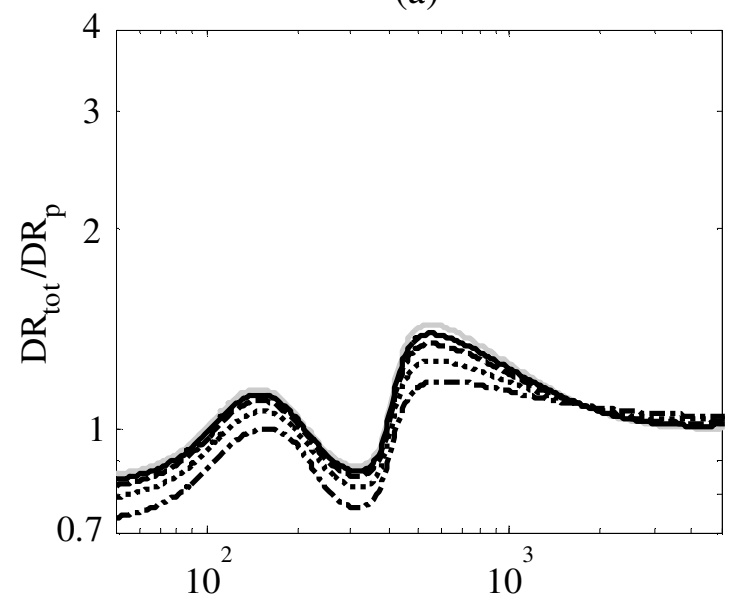

Frequency, $\mathrm{Hz}$ (b)

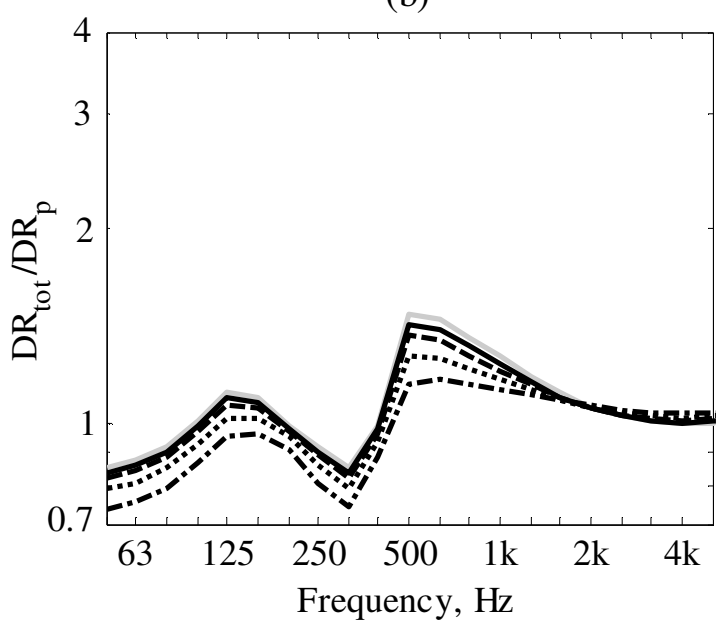

Figure 9: Ratio of total decay rate to decay rate of propagating wave of an infinite Timoshenko beam on a two layer foundation for various damping loss factors of the rail.,$- \eta=0.02 ;-, \eta=$ $0.1 ;---, \eta=0.2 ; \cdots \cdots, \eta=0.4 ;-\cdot-\cdot, \eta=0.8$. (a) Narrow-band; (b) one-third octave band.

\subsection{Decay rates of a finite length beam on two layer foundation}

Finally the same analysis is presented for a finite length Timoshenko beam on a double elastic foundation. This model is representative of a test track with the same properties 
as a real track but of finite length, as adopted to perform the measurements described in Section 2.2. The solution is found as in eq. (11) while the wavenumbers are those calculated from eq. (14).

The decay rate ratio is presented in Figure 10. This result is similar to that from the infinite beam on elastic foundation (Figure 9). Below $500 \mathrm{~Hz}$ in particular, the finiteness of the beam does not change the assessment of decay rates. Above this frequency, peaks occur in Figure 10(a) due to the existence of vibration modes; these are clearly visible for the loss factor equivalent to the undamped rail $(\eta=0.02)$ and are also present for higher loss factors up to 0.2. However, the effect of the modes disappears in a one-third octave band representation of the results as seen in Figure 10(b). At high frequency and for high loss factors the results drop below unity due to the grid spacing used close to the excitation point. A narrower spacing would give the same trend as in Figure 9 (for which a finer spacing is used) but the grid spacing adopted here is chosen to be consistent with the measurements of Section 2.2.

(a)

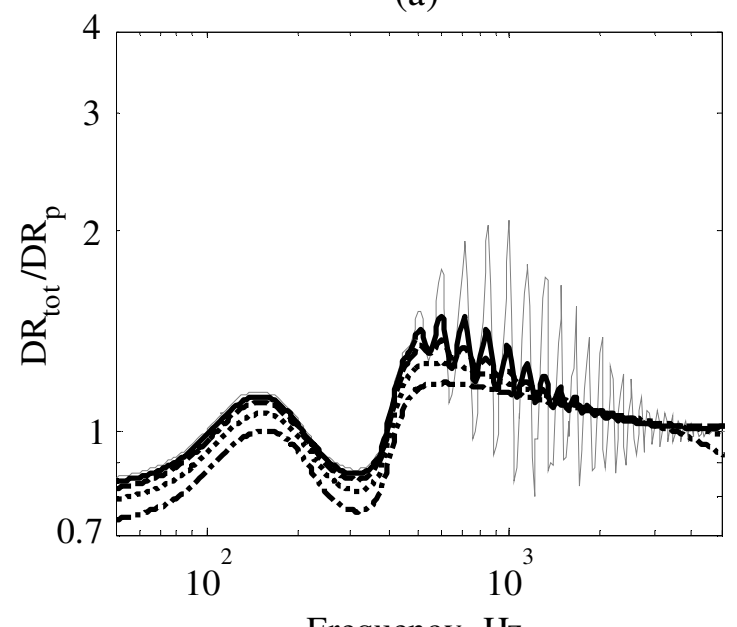

(b)

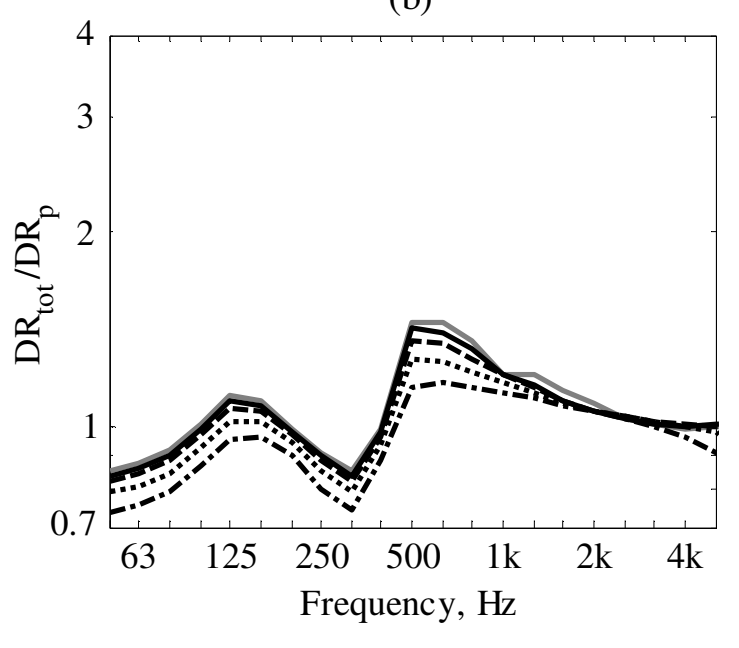

Figure 10: Ratio of total decay rate to decay rate of propagating wave of a finite length

Timoshenko beam on a two layer foundation for various loss factors.,$- \eta=0.02 ;-, \eta=0.1 ;--$ ,$- \eta=0.2 ; \cdots \ldots, \eta=0.4 ;-\cdot-\cdot, \eta=0.8$. (a) Narrow-band; (b) one-third octave band.

\subsection{Discussion}

Figure 11 summarises the results obtained in this section. The case of the infinite beam on double elastic foundation is omitted as its one-third octave band results are indistinguishable from those of the finite beam (32 m long) on elastic foundation. The case of the unsupported infinite beam (continuous line) gives the average trend of the unsupported finite beam (dash-dot line). This latter result can be used to comment on the applicability of the long rail method of Section 2.3. The highest ratio in one-third 
octave bands is found in this case to be 3.5 at $63 \mathrm{~Hz}$ for $\eta=0.1$. Between 125 and 2000 $\mathrm{Hz}$ it decreases from 1.5 to 1 . From eq. (1) it can be shown that this corresponds approximately to a change in noise radiated from the track of up to $1.7 \mathrm{~dB}$. The result for the beam supported on double elastic foundation (dashed line) shows that the measured decay rate accounting for all waves is within a factor of $0.7-1.4$ of the decay rate of the propagating wave. In terms of track radiated noise this can be approximated with an interval of $\pm 1.5 \mathrm{~dB}$.

(a)

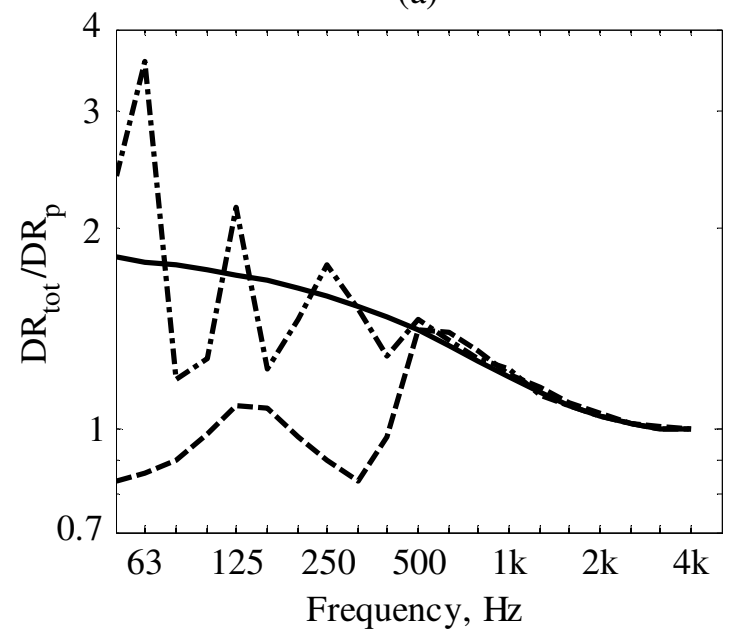

(c)

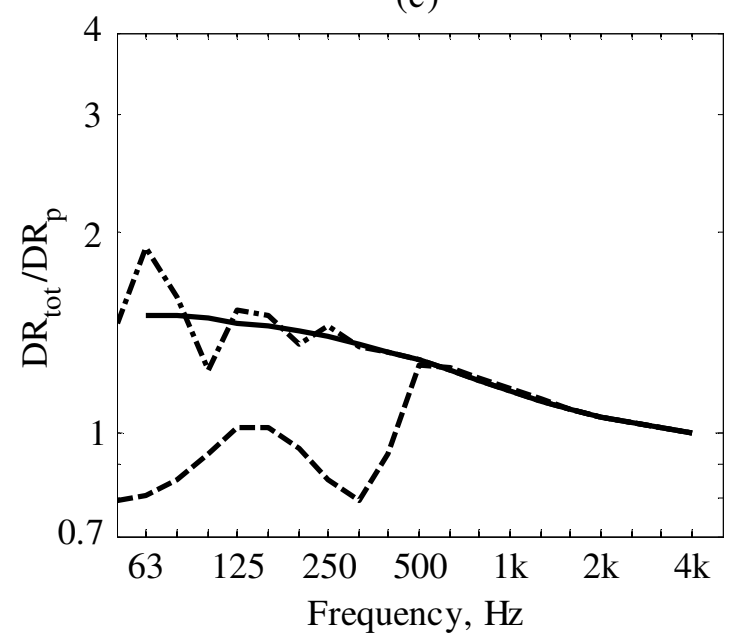

(b)

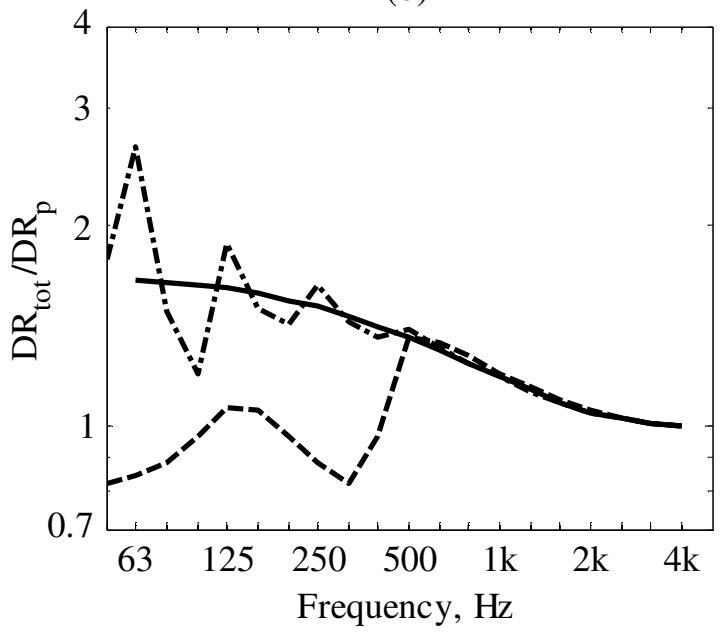

(d)

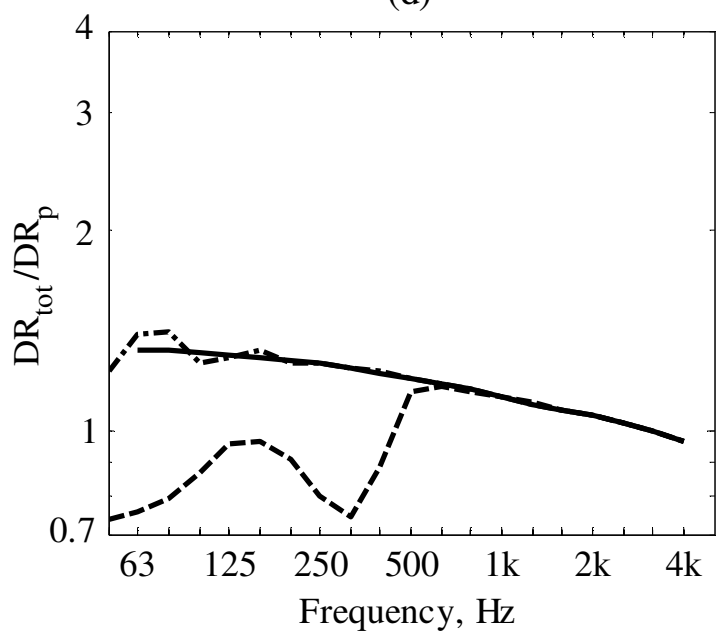

Figure 11: Decay rate estimated in one-third octave bands. -, Infinite unsupported Timoshenko beam; - - - ., finite length $(32 \mathrm{~m})$ unsupported Timoshenko beam; - - -, finite length $(32 \mathrm{~m})$

Timoshenko beam on a two-layer support. (a) $\eta=0.1$; (b) $\eta=0.2$, (c) $\eta=0.4$, (d) $\eta=0.8$.

As mentioned in the beginning of this section, when measuring decay rates on a real track according to the standard EN15461 [6], both propagating and evanescent waves are considered together. This also occurs on a test track, or on any finite length rail arrangement adopted to test rail dampers, where near field waves are captured; 
reflected waves also occur. The results presented so far show that the decay rates of the propagating wave differ from the total and a simple model can be adopted to quantify the difference. The applicability of these outcomes depends on the context. When the focus is on a generic assessment of a track prior to performing pass-by tests (see [27]), then decay rates measured according to [6] are a good indicator of the overall acoustic properties of the site. If the goal is instead to use measured decay rates as input into rolling noise models to improve their reliability, then the calculations shown in this section could be adopted to correct the measurement: the decay rates of the propagating wave can be extracted and used to update the imaginary part of this type of wave. Finally, if as in this paper, decay rates measured in different ways are to be compared with each other, it is important that the comparison is done in terms of the same quantity, which is chosen to be the decay rate of the propagating wave. These results will be shown in Section 5 after methods to measure decay rates of a damped short rail are introduced in the next section.

\section{Short free-rail methods for finding decay rates associated with dampers}

\subsection{Short rail measurements}

For a short section of damped rail, at low frequencies the FRF is dominated by clearly separated modes of vibration and distinct peaks can be observed. At higher frequencies, the damping values are generally higher and greater overlap between modes occurs, tending to make the response flatter and single modes less distinguishable. Because of this dynamic behaviour, two different procedures have been proposed for determining the decay rate of a damped free rail [15]. The first, considered in Section 4.2, is based on modal information ('modal method'), while the second, considered in Section 4.3, relies on the ratio between FRFs at the two ends of the rail ('direct method').

The length of the short rail used in the present work was chosen as $6 \mathrm{~m}$ as this gives a good compromise between practicality for tests in a laboratory and having sufficient measurable effect of the dampers with distance. In [15] measurements performed on a 4 $\mathrm{m}$ rail were also shown to provide a good estimation of the effect of dampers.

In the present tests ten dampers were installed at $0.6 \mathrm{~m}$ intervals symmetrically over a $6 \mathrm{~m}$ length of UIC 60 rail. The rail was supported at either end on stacks of rubber rail pads, resulting in a bounce mode of approximately $20 \mathrm{~Hz}$. Miniature accelerometers were mounted as close as possible to the ends of the rail (about $5 \mathrm{~mm}$ ). For both vertical and lateral measurements, the point FRF at one end and the transfer FRF to the other end 
were measured. Measurements were conducted in a laboratory with an ambient temperature of $20 \pm 2{ }^{\circ} \mathrm{C}$.

Figure 12 shows the mobility measured at either end of the $6 \mathrm{~m}$ free rail in the vertical and lateral directions with damper A fitted to the rail. For the vertical direction, in Figure 12(a), it is clearly possible to observe, at around $350 \mathrm{~Hz}$, the transition between the region dominated by individual modes and the region where the response is flatter due to the effect of the dampers. Below this frequency the point and transfer FRFs are similar in magnitude, whereas at higher frequencies there is a significant decay along the rail seen from the difference between point and transfer FRFs. The modal method will be applied only below $350 \mathrm{~Hz}$, while above this frequency the decay rates can be estimated by means of the direct method. For the lateral direction, in Figure 12(b), a similar distinction between the two frequency ranges can be drawn. However, in this direction, and with this particular type of dampers, another set of clearly distinguishable modes of the rail appears between around 800 and $2500 \mathrm{~Hz}$. Experimental modal analysis can be performed in this range and the modal method applied but, as the difference between the direct and transfer mobility is small, the direct method is not applicable.
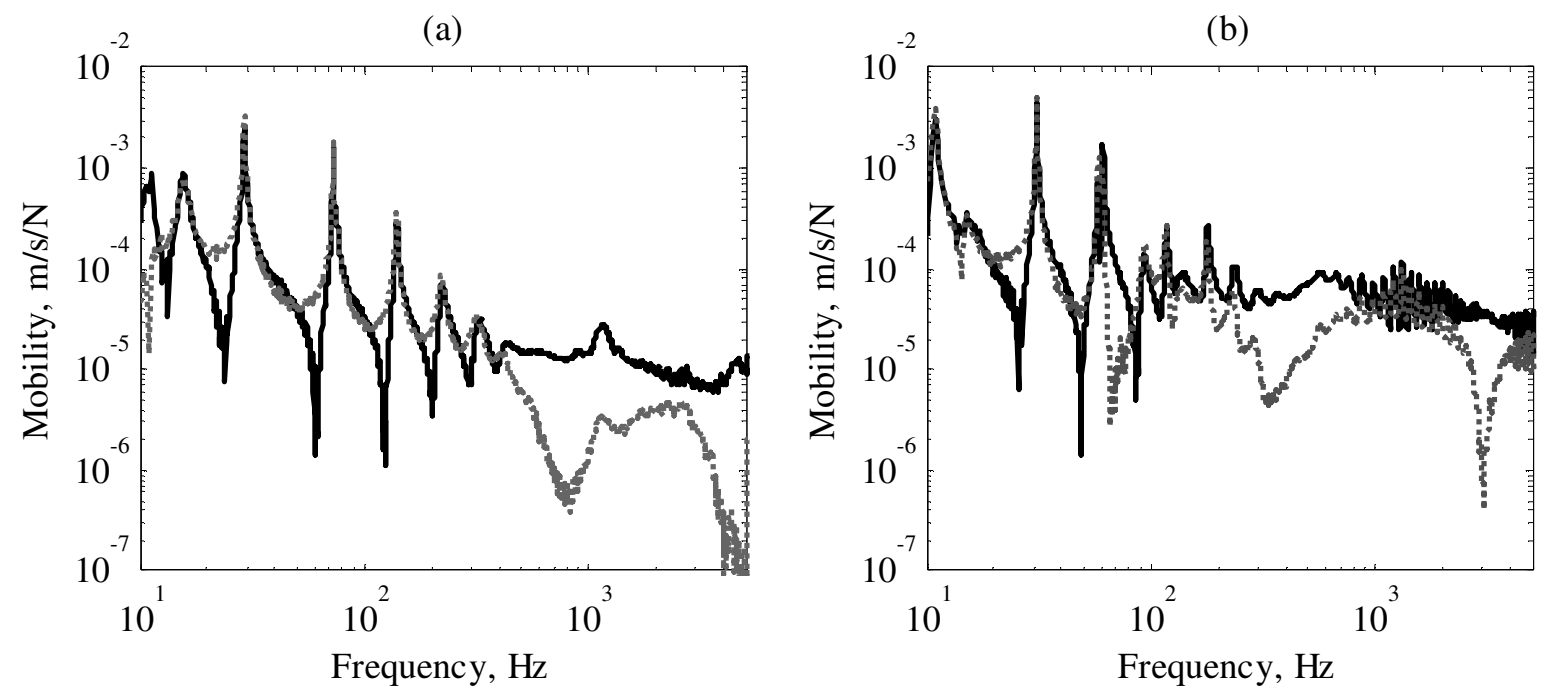

Figure 12: Example of driving point mobility ( - ) measured at one end and transfer mobility (......) measured at the other end of a damped $6 \mathrm{~m}$ long free rail. (a) vertical direction; (b) lateral direction

\subsection{Modal method}

According to the modal method the decay rates can be derived at the resonances from the natural frequencies and modal loss factors [15]. These can be identified through 
experimental modal analysis (e.g. see [28]). In addition, the velocity at which energy travels along the rail is required.

For a propagating wave of type $j$ (vertical, lateral, torsional, etc), the decay rate is given by [15]:

$D R_{j}=-10 \log _{10} e^{-\omega \eta / c_{j}^{g}}=4.343 \frac{\omega \eta}{c_{j}^{g}}$

where $\omega$ is the circular frequency, $\eta$ is the damping loss factor and $c_{j}^{g}$ is the group velocity. In the modal method, the damping loss factor is evaluated at the natural frequencies of the short rail. It remains, therefore, to determine the group velocity. In an undamped structure, the group velocity of a wave $j$ can be found as:

$c_{j}^{g}=\frac{\partial \omega}{\partial k_{j}}$

which represents the inverse slope of the dispersion curve (wavenumber $k$ versus circular frequency $\omega$ ). For a damped beam the wavenumber $k$ becomes complex and eq. (16) is no longer applicable [29]. Nevertheless, with the condition of low damping $(\eta<<1)$ eq. (16) can still be used to estimate the energy velocity by adopting the wavenumber of the undamped structure or the real part of the complex wavenumber. In this application the modal method could be applied for loss factor values smaller than about 0.05 , which makes eq. (15) still applicable with a negligible error.

The real part of the wavenumber can be estimated from the order of the mode, $n$, and the rail length, $L$. For vertical and lateral bending modes in a free-free beam it can be assumed that [30]:

$k_{n}=\frac{2 \pi}{\lambda_{n}} \approx\left(n+\frac{1}{2}\right) \frac{\pi}{L}$, for $n=1,2,3, \ldots$

where $\lambda_{n}$ is the wavelength of the $n$-th mode, while for torsional modes:

$k_{n}=\frac{2 \pi}{\lambda_{n}} \approx \frac{n \pi}{L}$, for $n=1,2,3, \ldots$

From this the dispersion curves can be obtained at the natural frequencies and the group velocity can be estimated from eq. (16). To assist in identifying the modal order $n$, the relative phase of the response at the two ends of the beam can be investigated. Further information could be obtained from additional response points. 
Following eqs. (17) and (18) the dispersion curves for vertical, lateral and torsional waves were obtained and are shown in Figure 13 for the rail fitted with the two different damper types (A and $\mathrm{B}$ ). To verify the procedure a finite element (FE) model of the free undamped rail was established as in [23]. Figure 13 also shows that the effect of the dampers at low frequency is mainly to add mass, which results in a reduction in the natural frequencies.

The gradient of the dispersion curve at each natural frequency was calculated as the first derivative of a $4^{\text {th }}$ order polynomial used to interpolate the dispersion curves. Results are given in Section 5.

(a)

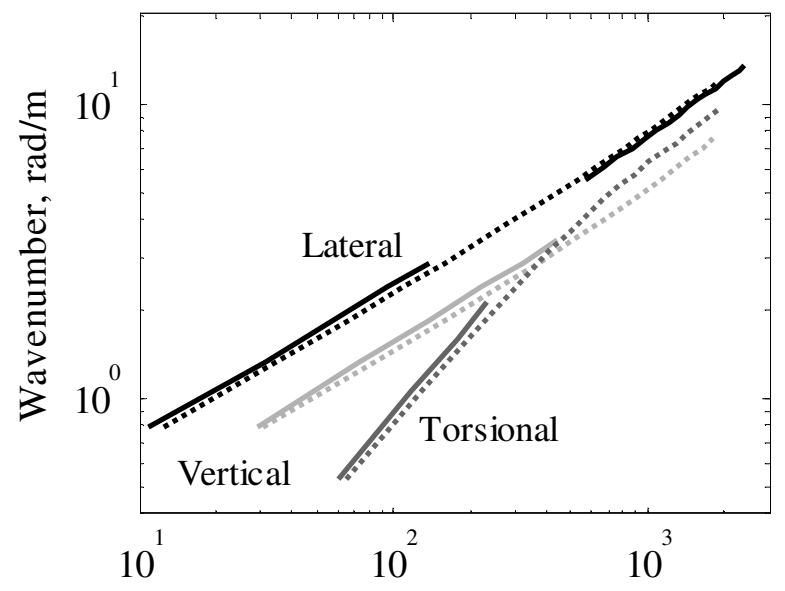

Frequency, $\mathrm{Hz}$ (b)

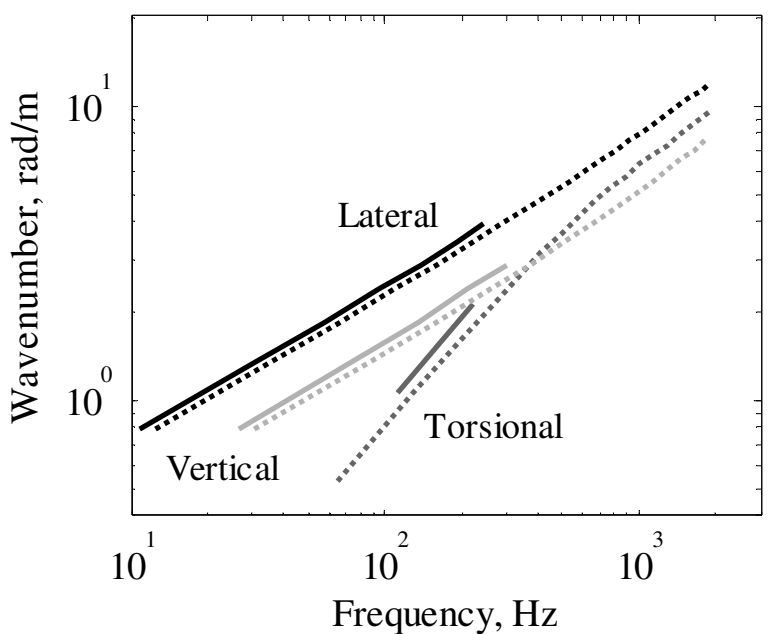

Figure 13: Dispersion curves estimated for a damped rail; (a) damper A; (b) damper B. —: estimated from experiments; …: from FE model.

\subsection{Direct method}

At higher frequencies where the modal overlap increases, the modal method can no longer be used and instead a direct method can be used for the short rail [15]. This is based on the measurement of FRFs at the two ends of the rail: the driving-point FRF at one end and transfer FRF at the other. In this case decay rates can be estimated as the decibel difference of the transfer FRF, $A\left(x_{1}\right)$, to the driving-point FRF $A\left(x_{0}\right)$, divided by the rail length $L$, in each one-third octave band $i$ :

$$
D R_{i}=\frac{1}{L} 20 \log _{10}\left(\frac{\left|A_{i}\left(x_{1}\right)\right|}{\left|A_{i}\left(x_{0}\right)\right|}\right)
$$

With low decay rates, the two FRFs are similar in magnitude, see Figure 12, and the relative error in $D R$ becomes large. By measuring the FRF at the ends of the rail evanescent waves and reflected waves are captured by the accelerometers as well as the 
directly propagating waves. This effect is quantified in the next section based on the Timoshenko beam model.

\subsection{Model of short rail methods - range of validity}

For a finite beam excited at one end, the displacement at any point along the beam is:

$u(x)=A_{1} e^{-i k_{e} x}+A_{2} e^{i k_{e} x}+A_{3} e^{i k_{p} x}+A_{4} e^{-i k_{p} x}$

where the wavenumbers $k_{p}$ and $k_{e}$ are defined in Section 3.

Wave amplitudes can be found by applying appropriate boundary conditions and eq. (20) can be used to calculate the decay rate as in eq. (19).

The result expected from the short rail direct method can be compared with the decay rate of a single propagating wave in an infinite beam (see eq. (7)) and the limitations of the proposed method analysed. Figure 14 shows the ratio between the decay rate estimated from the short rail direct method $\left(D R_{S R}\right)$ for a $6 \mathrm{~m}$ long free rail and the decay of the single propagating wave $\left(D R_{p}\right)$. Results for different loss factors of the rail are plotted against $D R_{p}$. This figure highlights first that the method is not applicable for low decay rate values; below $1 \mathrm{~dB} / \mathrm{m}$ the ratio fluctuates in an unacceptably wide range, even becoming negative. This is not surprising, as this corresponds to the modal range where the frequency response is dominated by a series of resonances and anti-resonances, as seen in Figure 12; above this range the direct short rail method can be successfully applied. For loss factors of at least 0.1 and decay rates between $1-2$ and $20 \mathrm{~dB} / \mathrm{m}$ all the lines tend to superimpose indicating a systematic small difference between the calculations with and without evanescent/reflected waves (see Figure 14(a)). At a decay rate of $2 \mathrm{~dB} / \mathrm{m}$ the ratio $D R_{S R} / D R_{p}$ is about 0.75 while at $5 \mathrm{~dB} / \mathrm{m}$ it is 0.9 ; it approaches unity for increasing decay rate. If converted into noise radiated from the track this would correspond to a difference of $1.2 \mathrm{~dB}$ at $2 \mathrm{~dB} / \mathrm{m}$, i.e. the short rail method would underpredict the benefit of a damper by this amount.

The direct short rail method should not be applied at relatively low values of the decay rate. In a $6 \mathrm{~m}$ long rail this limit is set at around $2 \mathrm{~dB} / \mathrm{m}$ but would decrease for longer rails. The range of applicability of the method can also be formulated in terms of the loss factor. Within the frequency range of interest of this application (up to $5 \mathrm{kHz}$ ) the minimum loss factor resulting in decay rates above $2 \mathrm{~dB} / \mathrm{m}$ is 0.05 . Again with a longer rail the lowest acceptable loss factor would be smaller. 
(a)

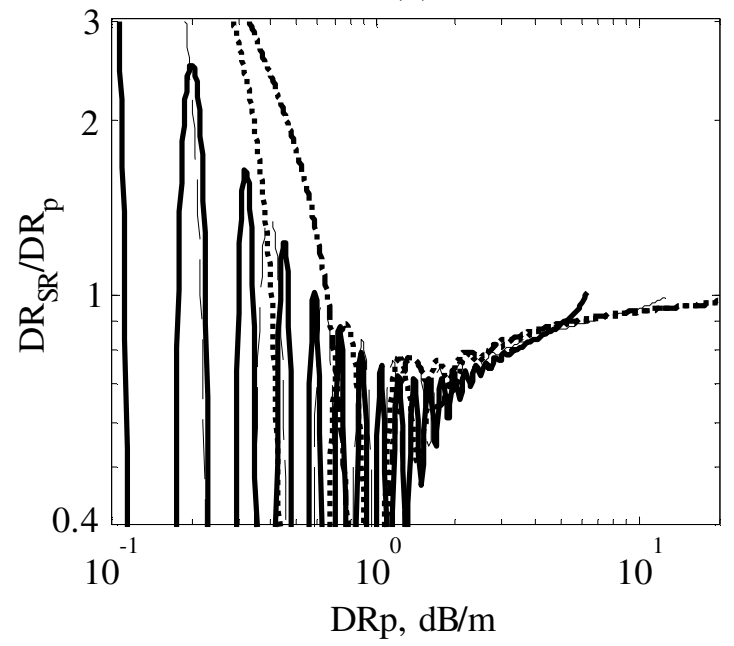

(b)

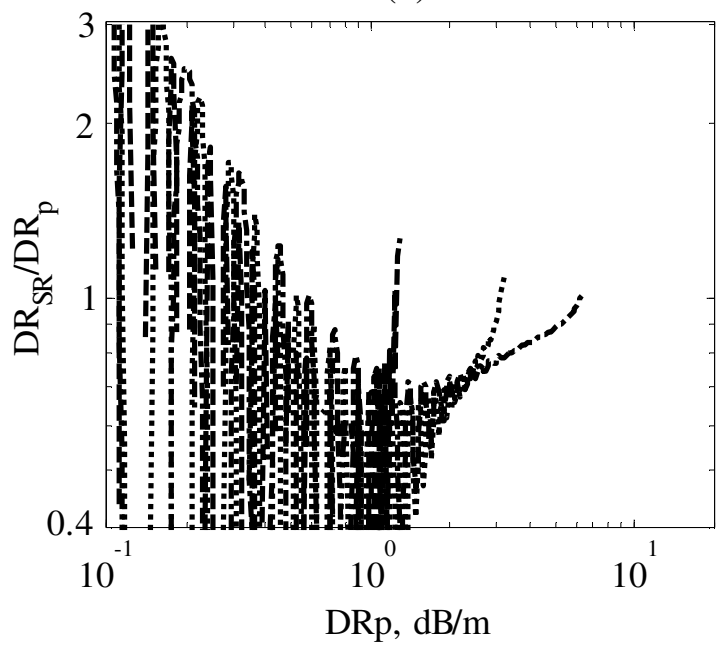

Figure 14: Effect of evanescent and reflected waves on the decay rate calculated as the ratio between vibration levels at the two ends of a free beam (short rail direct method). (a) results for high damping values,$- \eta=0.1 ;---, \eta=0.2 ; \cdots . ., \eta=0.4 ;-\cdot-\cdot, \eta=0.8$; (b) results for low damping values:,$--- \eta=0.02 ; \cdots \cdots, \eta=0.05 ;-\cdot-\cdot, \eta=0.1$.

\section{Comparison of results}

\subsection{Damped free-rail results}

This section compares decay rates measured on free rails according to the 'long rail method', 'short rail direct method' and 'modal method'. To allow a direct comparison among the three, the analytical calculations of Sections 3.2 and 4.4 can be adopted to remove the effect of evanescent and reflected waves from the 'long rail' and 'short rail direct' methods. As an example, Figure 15 shows the effect of applying these corrections to the vertical measurements of damper $\mathrm{B}$. These corrections are derived from the analyses shown in Section 3.2 for the long rail method and in Section 4.4 for the short rail method. In both cases the ratio $\mathrm{DR}_{\text {tot }} / \mathrm{DR}_{\mathrm{p}}$ is used to correct the measurements in order to obtain the decay rate of the propagating wave. Figure 15(a) shows the decay rate measured on the long rail before (dotted line) and after (solid line) corrections; a loss factor of $\eta=0.4$ is assumed to calculate these corrections (see Figure 8) although the corrections are fairly insensitive to the loss factor. As a result the decay rates are slightly lowered for frequencies below $1 \mathrm{kHz}$. For the short rail method the corrections have been shown to depend on the measured decay value itself, see Figure 14, and result in an increase in the decay rate at all frequencies. 
(a)

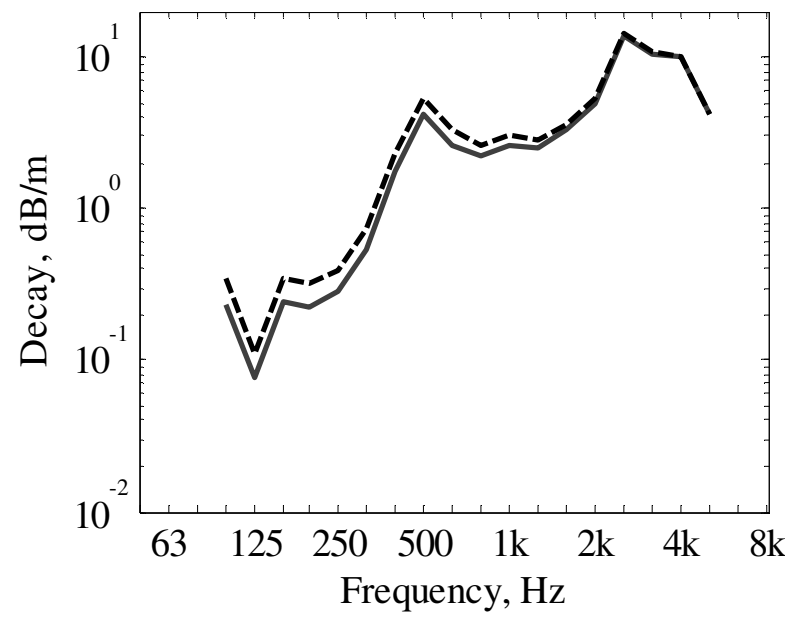

(b)

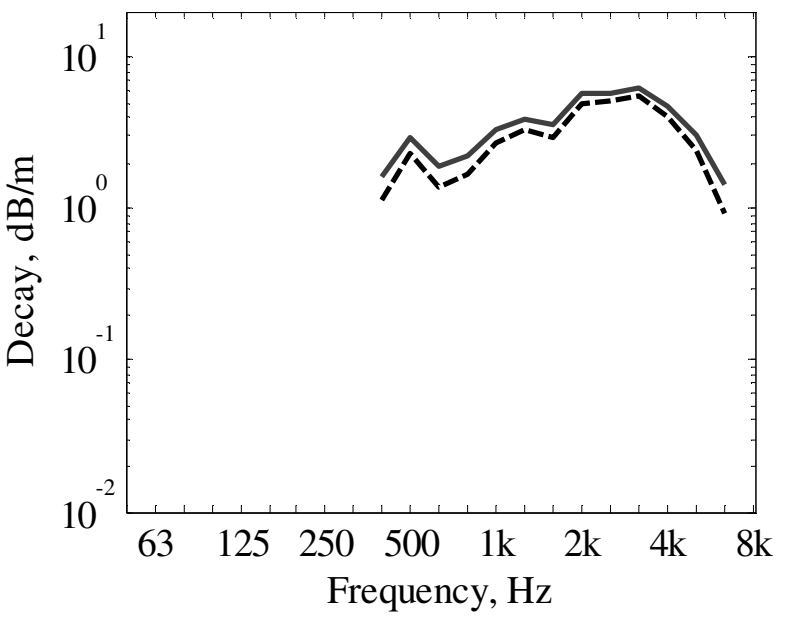

Figure 15. Effect of correcting measured vertical decay rate of damper B to remove the reflected/evanescent waves. (a) Long rail method, (b) Short rail direct method. -.--, Non-corrected measurements; - , propagating wave only.

The vertical decay rates for dampers A and B determined according to the various methods and corrected to account for near field and end effects are given in Figure 16. As discussed in Section 4, the ranges of applicability of the two short rail methods are complementary so that they give results in different parts of the frequency range. Moreover, the results appear consistent where the two ranges meet.

It can be observed from the results in Figure 16 that the level of agreement between the long rail method and the short rail direct method is on average quite good although there are discrepancies of up to a factor of 4 for damper $\mathrm{A}$ and up to factor of 3 for damper $\mathrm{B}$ (considering frequencies above $200 \mathrm{~Hz}$ ). However there are several potential sources of discrepancy between the long rail and short rail results. First, the way the dampers are attached to the rail may not be entirely repeatable. Second, there were differences in temperature as the measurements on the long rail were performed outdoors whereas the short rail ones were performed in a laboratory. This can affect the damper performance due to the sensitivity of the elastomeric materials to temperature [31]. The temperature of each test is reported in the corresponding figures and differences of up to $10^{\circ} \mathrm{C}$ can be noted. The extent of this effect will vary according to the damper design. 
(a)

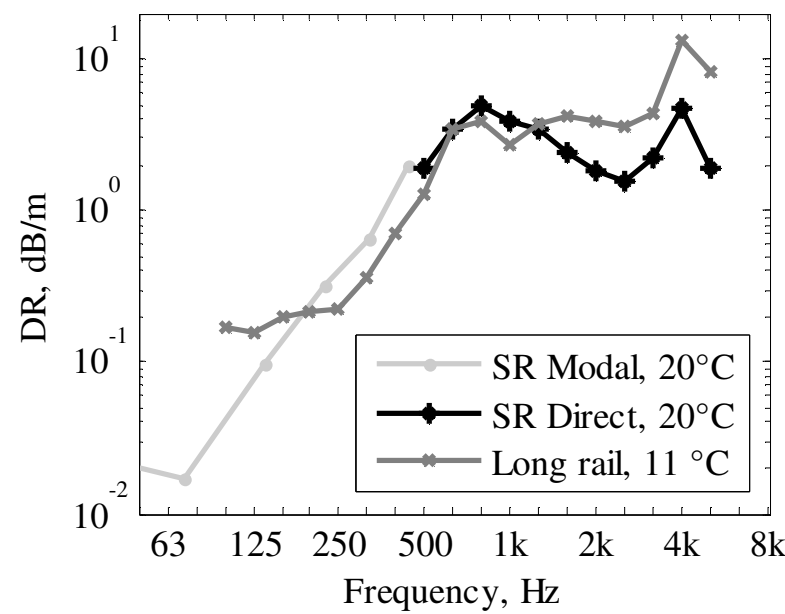

(b)

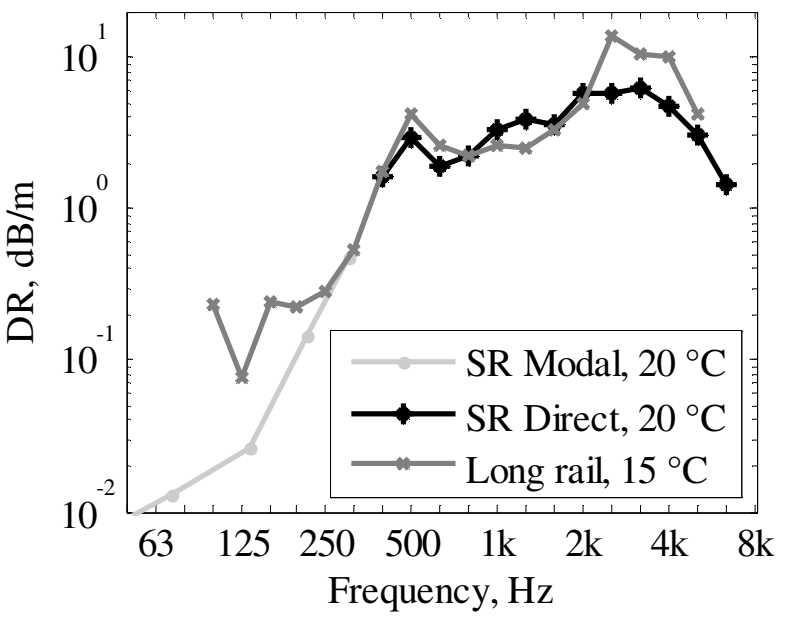

Figure 16: Decay rate of free rail in vertical direction, comparison among the three methods proposed: 'short rail modal method' (SR modal), 'short rail direct method' (SR direct) and 'long rail method'. (a) damper A, (b) damper B.

Decay rates for the lateral direction are shown in Figure 17. Again, a good continuity is found between the two short rail methods. The comparison between the long rail method and the short rail direct method shows differences of up to a factor of 2-3. The agreement is better for damper B than for damper A. Again the temperature difference can be in part responsible for the differences found. Overall the results from the short rail direct method are lower than those from the long rail method even after applying corrections.

(a)

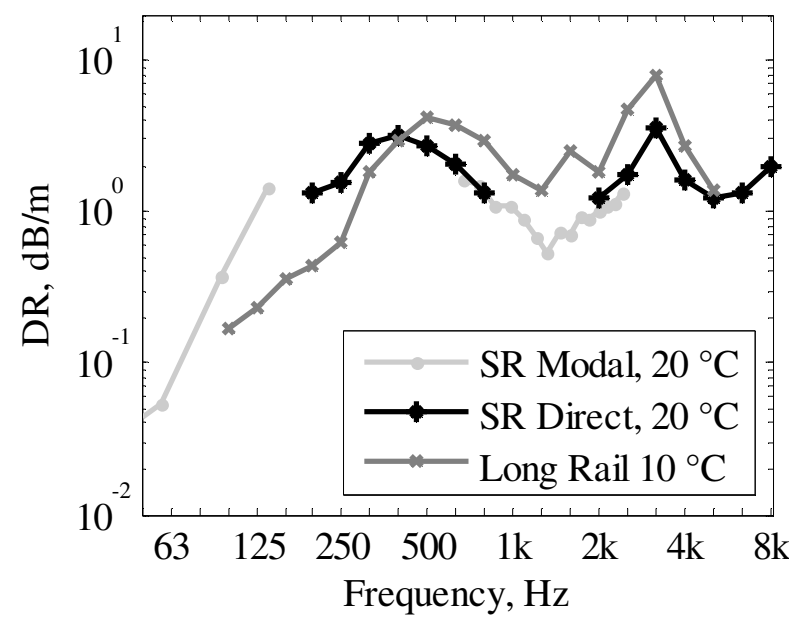

(b)

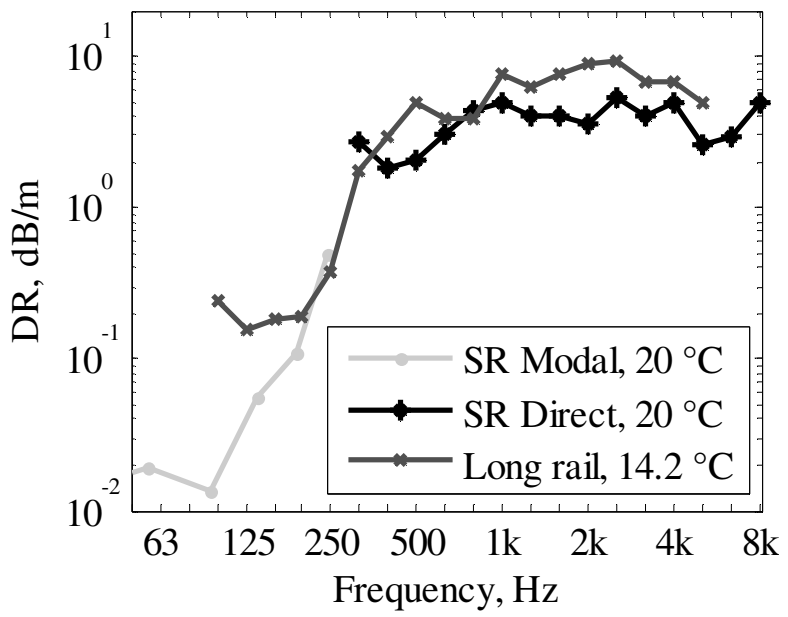

Figure 17: Decay rates of free rail in lateral direction, comparison among the three methods proposed: 'short rail modal method' (SR modal), 'short rail direct method' (SR direct) and 'long rail method'. (a) damper A; (b) damper B.

The modal method is only applicable in the frequency range characterised by the responses of individual modes. For 4 or $6 \mathrm{~m}$ long rails this corresponds to the low 
frequency range where the dampers are not intended to be effective. Moreover, the method requires some additional work in terms of modal identification which becomes harder as the effect of the dampers increases. Although the modal method is practicable in principle, it only works in those frequency ranges where the estimated decay rates would be small and their impact on those of the track would be negligible. In practice, the short rail direct method is easier to apply and it works in the frequency range where the dampers are effective.

\subsection{Track decay rate validation measurements}

To verify further the reliability of the free rail methods the decay rates of the damped track estimated from the combination of either the short or long rail measurements with those measured on the undamped track are compared in Figure 18 and Figure 19 with the results directly measured on the same test track fitted with dampers, see Section 2.2. Considering the conclusions drawn above about the modal method, this is not considered further in this section. As the effect of applying corrections to the free rail methods has been shown to introduce some improvement in the agreement between different methods these are also included in this section. The effect of including or disregarding these corrections on the predicted noise is discussed below in Section 6 .

Figure 18 shows results for damper A for vertical and lateral directions; Figure 19 is devoted to damper B. In both cases and for both directions, the effect of adding dampers has been estimated reasonably well by means of the free rail methods. The short rail method tends to underestimate the decay rate while the 'long rail' result is either above or nearly coincident with the measurements on the damped track. Changes in temperature are also in this case responsible for some of the differences among the results.

Including the corrections to account for evanescent and reflected waves results in some benefit as the decay rates measured with different methods are in better agreement after applying corrections. The short rail method applied without any additional correction would by itself provide a lower bound for track decay rate estimation, which corresponds to an upper bound for the track radiated noise. This can potentially become a reliable method to be used in assessing damper effectiveness without the need of installation on the track. If more accurate analysis is required and the corrections are to be included, it should be noted that the effect of reflected waves depends also on the length of the rail and the results shown in this paper are valid only for a $6 \mathrm{~m}$ long rail. In general, however, if a longer rail is used the corrections will be smaller. 
(a)

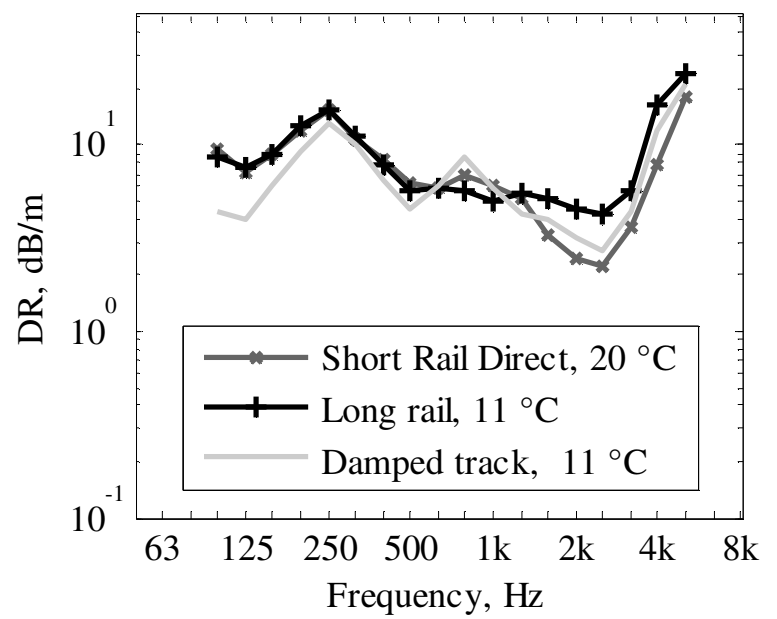

(b)

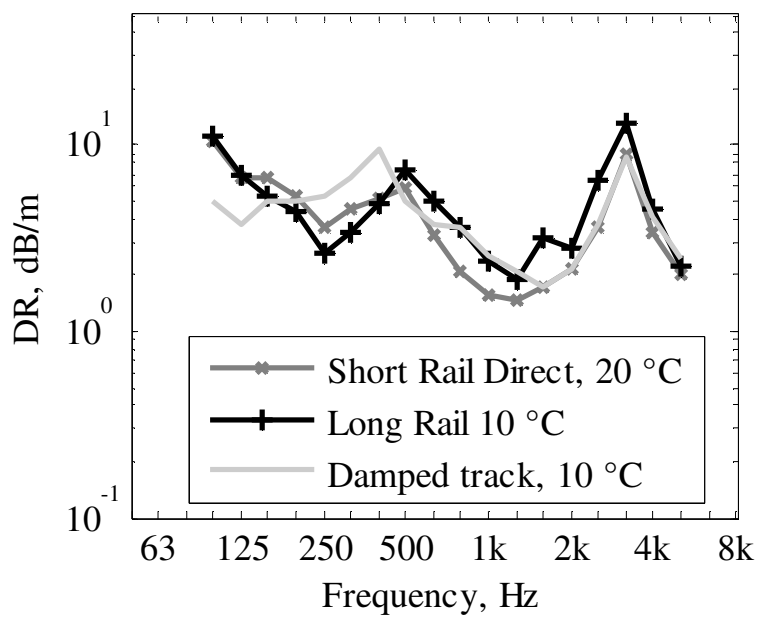

Figure 18: Decay rate measured on a track (damped track) for damper A compared with total decay rate estimated according to the 'short rail direct method' and the 'long rail method'. (a) vertical; (b) lateral.

(a)

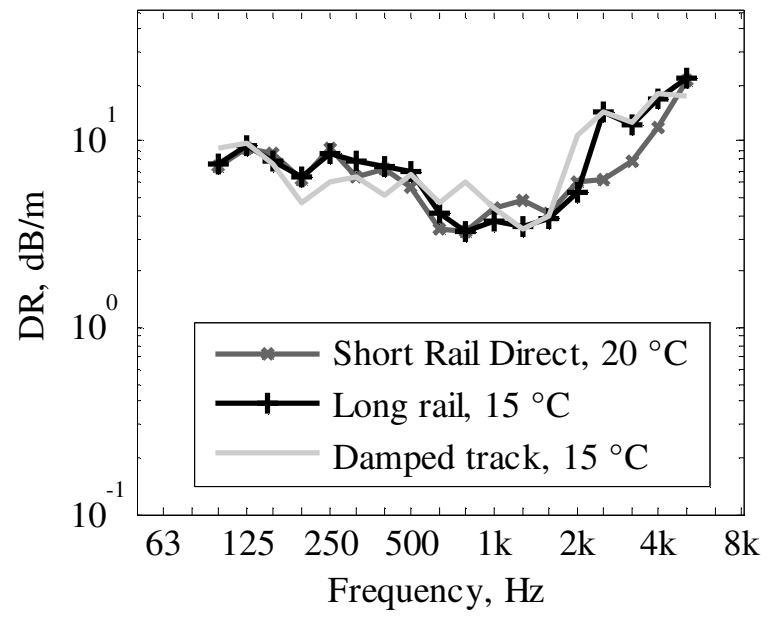

(b)

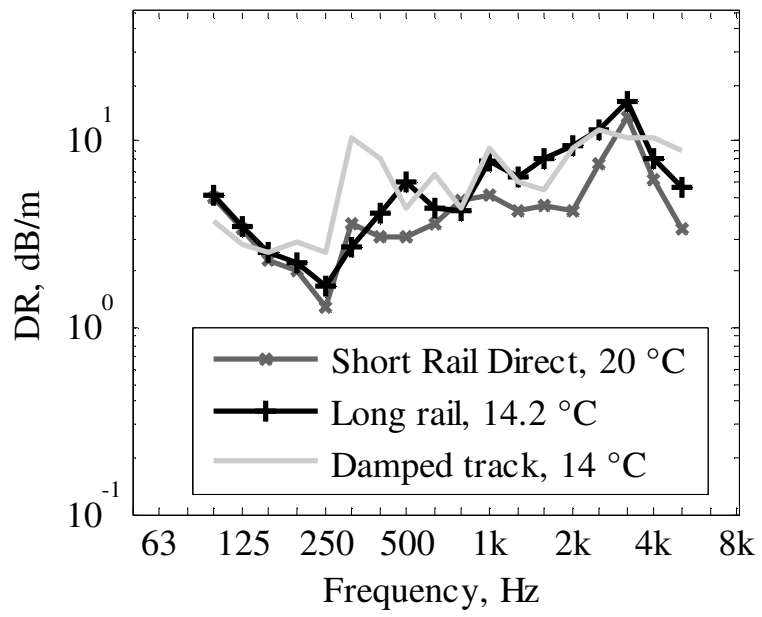

Figure 19: Decay rate measured on a track (damped track) for damper B compared with total decay rate estimated according to the 'short rail direct method' and the 'long rail method'. (a) vertical; (b) lateral.

\section{Predictions of damper effectiveness}

Decay rates measured using the short rail direct method have been used to predict [20, $21]$ the potential effect of dampers on pass-by noise and to quantify the final effect of correcting decay rates as in Section 4.4. Decay rates below $250 \mathrm{~Hz}$ were left unmodified due to measurement uncertainty and the general ineffectiveness of the dampers at these low frequencies. The most important parameters used in the model are summarised in Appendix A. A wheel modal model corresponding to a typical regional train has been used (diameter equal to $900 \mathrm{~mm}$ ), with roughness representative for disc braked wheels 
[32]. Track parameters are selected to be representative of the test track (see Section 2.2) and the rail roughness is assumed to follow the limit curve defined in [27]. The relatively low pad stiffness $(120 \mathrm{MN} / \mathrm{m})$ makes this track a good candidate for the application of rail dampers, with the contribution from rail noise expected to be predominant in the region where the dampers are effective, i.e. 400-2000 Hz.

Figure 20(a) shows the sound power predicted for an undamped track, using measured decay rates from Figure 2, and with a train speed of $120 \mathrm{~km} / \mathrm{h}$. The noise contribution of the rail is dominant in the mid-frequency region and, in particular, the noise due to vertical vibration is greater than that due to lateral vibration in the whole frequency range. Wheel noise is the main source at high frequency, while the contribution of the sleeper is at a much lower level since the low stiffness pads tend to isolate the sleeper. Figure 20(b) presents corresponding sound power levels after introducing decay rates from the short rail direct method to represent a damped track (damper B). In Figure 20(b) the rail contribution has been reduced significantly due to the effect of the dampers. Both vertical and lateral rail components have been affected, with a respective decrease in sound power level of 6.1 and $10 \mathrm{~dB}$. For this example the overall acoustic power has been reduced by $3.7 \mathrm{~dB}$.

(a)

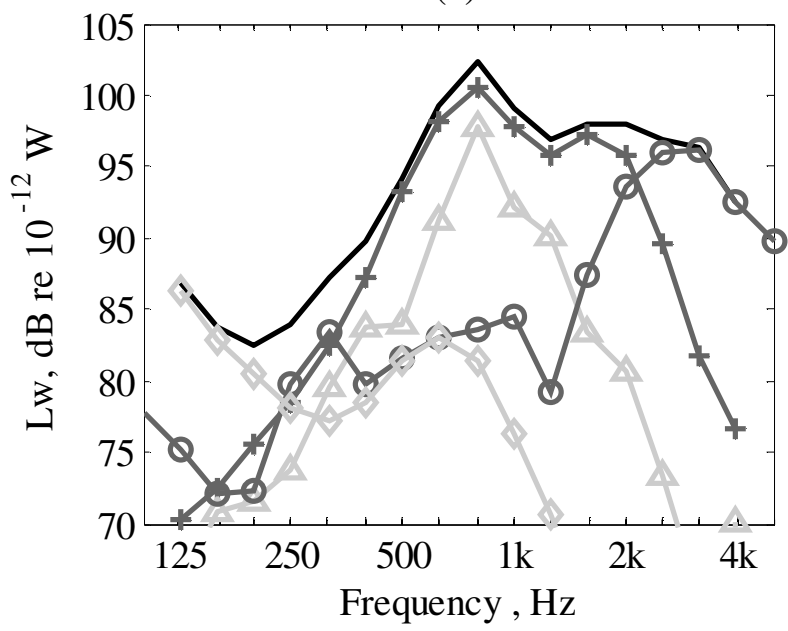

(b)

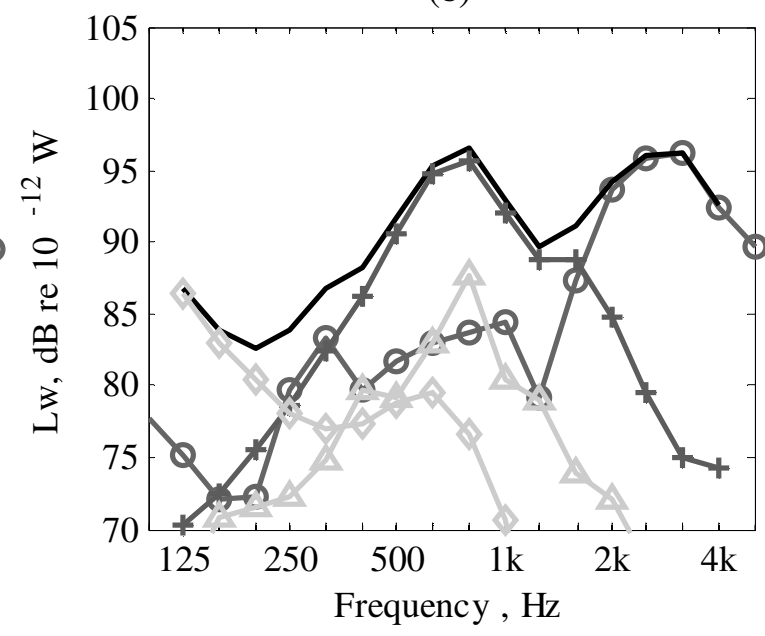

Figure 20: Sound power levels predicted (a) on undamped track and (b) on damped track, equipped with soft pads $(120 \mathrm{MN} / \mathrm{m})$ for train speed of $120 \mathrm{~km} / \mathrm{h} . \triangleleft$ : sleeper; $\_$: rail lateral direction; — : rail vertical direction; ——: wheel; — : total.

The effect of dampers on the overall noise level depends on the relative importance of each component prior to installation of the dampers. On a different track with different pad stiffness and possibly a different wheel type, the same type of dampers will have a different effect. As an example calculations have been made by changing pad stiffness to 
$800 \mathrm{MN} / \mathrm{m}$. The predicted total reduction in overall sound power is only $1.6 \mathrm{~dB}$; the reduction in all the track components considered together (rail vertical, rail lateral and sleeper) is $4.2 \mathrm{~dB}$.

To verify how much the corrections described in Section 4.4 affect the noise predictions, the case for soft pads has been also calculated with non-corrected decay rates. In this case the presence of dampers is predicted to reduce the track component (rail and sleeper) by $5.8 \mathrm{~dB}$, against $6.5 \mathrm{~dB}$ calculated with corrections; the corresponding total reductions are $3.4 \mathrm{~dB}$ and $3.7 \mathrm{~dB}$.

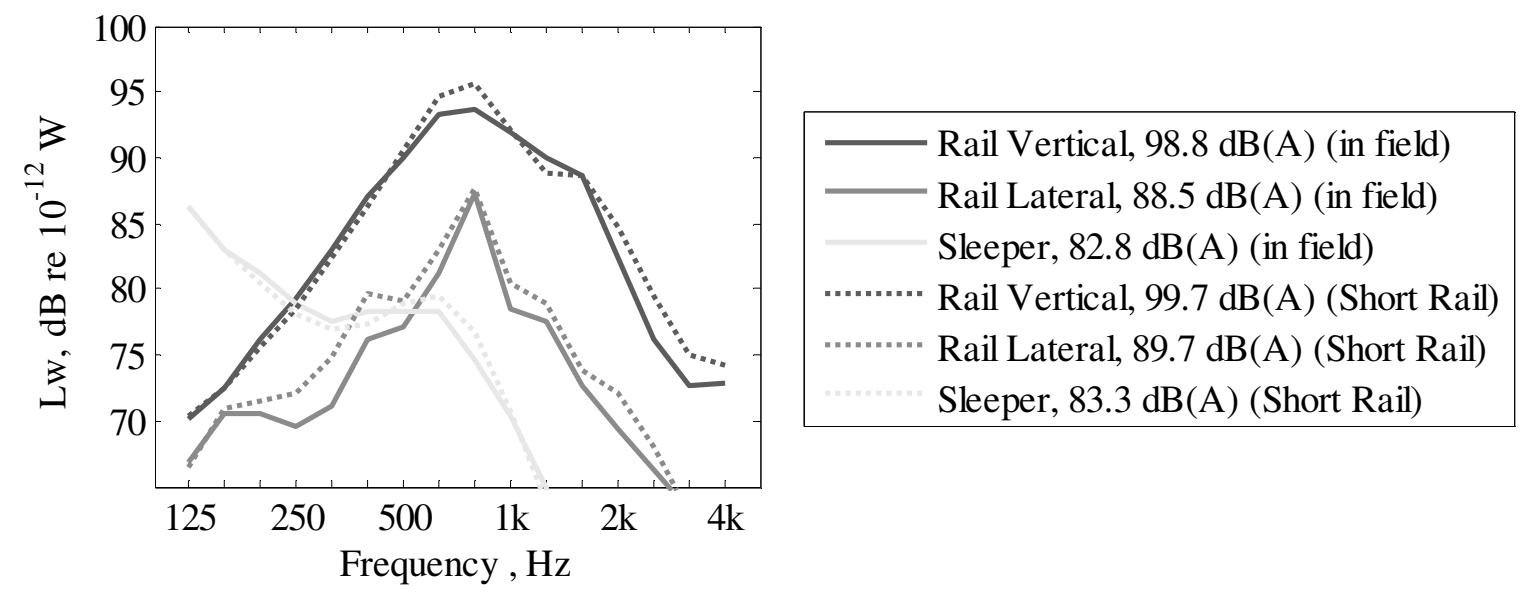

Figure 21. Sound power level for track components only. Comparison between results based on track decay rates measured in field and using short rail method. Pad stiffness $120 \mathrm{MN} / \mathrm{m}$, speed $120 \mathrm{~km} / \mathrm{h}$.

Finally the noise is also predicted with the track decay rate measured directly on the test track fitted with dampers (Figure 2). This will verify the sensitivity of noise predictions to the differences shown in Figure 18 and Figure 19. In this case the track components of noise are reduced by $7.3 \mathrm{~dB}$ and the total power is reduced by $4.0 \mathrm{~dB}$. Compared with the results from the short rail method including the corrections on decay rates, the change in overall noise differs by $0.3 \mathrm{~dB}$ and the track components by $0.8 \mathrm{~dB}$. Figure 21 shows sound power spectra radiated by each track component. The most important gap is found in the vertical direction at $800 \mathrm{~Hz}$ where the two predictions differ by $2 \mathrm{~dB}$ (see also Figure 19(a)). Overall the difference is equal to $0.9 \mathrm{~dB}(\mathrm{~A})$ in the vertical direction and 1.2 $\mathrm{dB}(\mathrm{A})$ in the lateral. The differences are larger compared with the short rail method without any correction; however as such an approach would underestimate the effect of dampers it can still be used to give an upper limit for noise. 


\section{Conclusions}

Experimental methods have been proposed for determining the effectiveness of rail dampers without the need to mount them on an operational track. Three methods for measuring decay rates of damped freely supported rails have been tested with two different types of damper and they are found to be in reasonable agreement between 300 $\mathrm{Hz}$ and $5 \mathrm{kHz}$. The modal method for determining decay rates was generally restricted in practice to low frequencies $(<300 \mathrm{~Hz})$. As the dampers are found to have little influence on the overall track decay rates below $400 \mathrm{~Hz}$, the direct short-rail method, yielding good results down to $300 \mathrm{~Hz}$, is considered to be sufficient for most applications.

In the vertical direction, between $800 \mathrm{~Hz}$ and $2 \mathrm{kHz}$, the short rail direct method and the long rail method show acceptable agreement. In comparing results from the short and long rails several possible causes of discrepancies have been considered. The most important are likely to be the repeatability of damper installation, which depends on the way each damper type is designed, the effect of temperature, and end effects due to the finite rail lengths. Measuring the free rail in a controlled environment would remove the effects of temperature. The short rail test method is therefore preferred for reasons of practicality.

Models of the transmission of vibration in the rail have been used to quantify the influence of end effects and of evanescent waves at the forcing point. It is shown however that, in the presence of dampers, the end effects and evanescent waves have a marginal contribution on the overall estimation of damper effectiveness; their effect on the overall predicted noise is estimated to be less than $0.5 \mathrm{~dB}$ in the examples considered.

Vertical and lateral decay rates of a damped track predicted from the results of the undamped track and from the free rail methods agree quite well with direct measurements of the damped track carried out for validation of the method, although there are some differences. However, in this case, the effect of changes in temperature on the pad stiffness is another source of variability that contributes to the dispersion of the results. Nevertheless the proposed laboratory test method appears to be suitable to form a standardized method for assessing different rail dampers.

\section{Acknowledgments}

The authors would like to acknowledge the assistance of the STARDAMP partners: Alstom, Deutsche Bahn, GHH-Valdunes, Schrey \& Veit, SNCF, Tata Steel, TU Berlin and Vibratec and the funding from the Deufrako cooperation (German funding by 
BMWI; FKZ 19U10012 A, B, C, D; French funding by FUI). Part of the work described has been supported by the EPSRC under the programme grant EP/H044949/1, 'Railway Track for the $21^{\text {st }}$ Century (Track21)'. All data published in this paper are openly available from the University of Southampton repository at doi:10.5258/SOTON/379558. 


\section{Appendix A. Numerical parameters}

Table A 1. Parameters used for analytical calculation.

\begin{tabular}{ll}
\hline Wheel parameters & \\
\hline Wheel load (kN) & 50 \\
Vehicle length (m) & 26 \\
Wheel type & LK900 \\
Wheel radius (m) & 0.45 \\
\hline Rail parameters & \\
\hline Vertical bending stiffness $\left(\mathrm{Nm}^{2}\right)$ & $6.42 \times 10^{6}$ \\
Lateral bending stiffness $\left(\mathrm{Nm}^{2}\right)$ & $1.07 \times 10^{6}$ \\
Density (kg/m $\left.{ }^{3}\right)$ & 7850 \\
Rail mass per length (kg/m) & 60 \\
Vertical and lateral shear coefficient & 0.4 \\
Rail vertical and lateral loss factor & 0.02 \\
\hline Pad parameters & \\
\hline Pad vertical stiffness (MN/m) & 120 \\
Pad lateral stiffness (MN/m) & 13 \\
Pad vertical and lateral loss factor & 0.2 \\
\hline Sleeper parameters & \\
\hline Sleeper mass (kg) & 280 \\
Sleeper Young's modulus (GPa) & 41.3 \\
Sleeper Poisson's ratio & 0.15 \\
Sleeper shear coefficient & 0.83 \\
Sleeper density (kg/m $\left.{ }^{3}\right)$ & 2750 \\
Sleeper loss factor & 0.02 \\
Sleeper length (m) & 2.6 \\
\hline
\end{tabular}




\section{References}

[1] D.J. Thompson, B. Hemsworth, N. Vincent, Experimental validation of the TWINS prediction program for rolling noise, Part 1: description of the model and method, Journal of Sound and Vibration, 193 (1996) 123-135.

[2] D.J. Thompson, P. Fodiman, H. Mahé, Experimental validation of the TWINS prediction program for rolling noise, part 2: Results, Journal of Sound and Vibration, 193 (1996) 137-147.

[3] C.J.C. Jones, D.J. Thompson, Extended validation of a theoretical model for railway rolling noise using novel wheel and track designs, Journal of Sound and Vibration, 267 (2003) 509-522.

[4] P.J. Remington, Wheel/rail noise-Part IV: Rolling noise, Journal of Sound and Vibration, 46 (1976) 419-436.

[5] D. Thompson, Railway Noise and Vibration: Mechanisms, Modelling and Means of Control, Elsevier Science, 2009.

[6] EN 15461:2008. Railway applications - Noise emissions, Characterization of the dynamic properties of track sections for pass by noise measurements.

[7] C.J.C. Jones, D.J. Thompson, R.J. Diehl, The use of decay rates to analyse the performance of railway track in rolling noise generation, Journal of Sound and Vibration, 293 (2006) 485-495.

[8] M.H.A. Janssens, M.G. Dittrich, F.G. de Beer, C.J.C. Jones, Railway noise measurement method for pass-by noise, total effective roughness, transfer functions and track spatial decay, Journal of Sound and Vibration, 293 (2006) 1007-1028.

[9] N. Vincent, P. Bouvet, D.J. Thompson, P.E. Gautier, Theoretical optimization of track components to reduce rolling noise, Journal of Sound and Vibration, 193 (1996) 161-171.

[10] D.J. Thompson, P.E. Gautier, Review of research into wheel/rail rolling noise reduction, Proceedings of the Institution of Mechanical Engineers, Part F: Journal of Rail and Rapid Transit, 220 (2006) 385-408.

[11] J. Oertli, The STAIRRS project, work package 1: a cost-effectiveness analysis of railway noise reduction on a European scale, Journal of Sound and Vibration, 267 (2003) 431-437.

[12] P. van den Dool, Rail dampers, rail infrastructure gets quiet, in: INTER-NOISE and NOISECON Congress and Conference Proceedings, 2007, pp. 2312-2320.

[13] J. Maes, H. Sol, A double tuned rail damper-increased damping at the two first pinned-pinned frequencies, Journal of Sound and Vibration, 267 (2003) 721-737.

[14] T.X. Wu, H.P. Liu, Reducing the rail component of rolling noise by vibration absorber: theoretical prediction, Proceedings of the Institution of Mechanical Engineers, Part F: Journal of Rail and Rapid Transit, 223 (2009) 473-483.

[15] D.J. Thompson, C.J.C. Jones, T.P. Waters, D. Farrington, A tuned damping device for reducing noise from railway track, Applied Acoustics, 68 (2007) 43-57.

[16] E. van Haaren, G.A. van Keulen, New Rail Dampers at the Railway Link Roosendaal-Vlissingen Tested within the Dutch Innovation Program, in: B. Schulte-Werning, D. Thompson, P.-E. Gautier, C. Hanson, B. Hemsworth, J. Nelson, T. Maeda, P. Vos (Eds.) Noise and Vibration Mitigation for Rail Transportation Systems, Springer Berlin Heidelberg, 2008, pp. 378-383.

[17] B. Asmussen, D. Stiebel, P. Kitson, D. Farrington, D. Benton, Reducing the Noise Emission by Increasing the Damping of the Rail: Results of a Field Test, in: B. Schulte-Werning, D. Thompson, P.E. Gautier, C. Hanson, B. Hemsworth, J. Nelson, T. Maeda, P. Vos (Eds.) Noise and Vibration Mitigation for Rail Transportation Systems, Springer Berlin Heidelberg, 2008, pp. 229-235.

[18] B. Asmussen, STARDAMP Standardisation of damping technologies for the reduction of rolling noise - Final Report. Deufrako Project STARDAMP, in, 2013. 
[19] D.J. Thompson, C.J.C. Jones, N. Turner, Investigation into the validity of two-dimensional models for sound radiation from waves in rails, The Journal of the Acoustical Society of America, 113 (2003) 1965-1974.

[20] M.G.R. Toward, G. Squicciarini, D.J. Thompson, Y. Gao, Estimating the Performance of Rail Dampers Using Laboratory Methods and Software Predictions, in: J. Nielsen, D. Anderson, P.-E. Gautier, M. Iida, J. Nelson, D. Thompson, T. Tielkes, D. Towers, P. de Vos (Eds.) Noise and Vibration Mitigation for Rail Transportation Systems, Springer Berlin Heidelberg, 2015, pp. 47-54.

[21] B. Betgen, P. Bouvet, G. Squicciarini, D.J. Thompson, C.J.C. Jones, Estimating the Performance of Wheel Dampers Using Laboratory Methods and a Prediction Tool, in: J. Nielsen, D. Anderson, P.E. Gautier, M. Iida, J. Nelson, D. Thompson, T. Tielkes, D. Towers, P. de Vos (Eds.) Noise and Vibration Mitigation for Rail Transportation Systems, Springer Berlin Heidelberg, 2015, pp. 39-46.

[22] G. de France, Railway track: effect of rail support stiffness on vibration and noise, University of Southampton, 1998.

[23] D.J. Thompson, Experimental analysis of wave propagation in railway tracks, Journal of Sound and Vibration, 203 (1997) 867-888.

[24] T.X. Wu, D.J. Thompson, Analysis of lateral vibration behavior of railway track at high frequencies using a continuously supported multiple beam model, 106 (1999) 1369-1376.

[25] T.X. Wu, D.J. Thompson, Application of a multiple-beam model for lateral vibration analysis of a discretely supported rail at high frequencies, 108 (2000) 1341-1344.

[26] K. Graff, Wave Motion in Elastic Solids (Dover Books on Physics), Dover Publications, 1991.

[27] ISO 3095:2013, Acoustics - Railway applications - Measurements of noise emitted by railbound vehicle, International Standard Organisation.

[28] D.J. Ewins, Modal Testing: Theory, Practice and Application (Mechanical Engineering Research Studies: Engineering Dynamics Series), 2nd ed., Wiley, 2003.

[29] M. Hayes, M.J.P. Musgrave, On energy flux and group velocity, Wave Motion, 1 (1979) 75-82.

[30] F. Fahy, P. Gardonio, Sound and Structural Vibration, Transmission and Response, 2nd ed., Academic Press, 2007.

[31] N. Ahmad, D.J. Thompson, C.J.C. Jones, A.H. Muhr, Predicting the effect of temperature on the performance of elastomer-based rail damping devices, Journal of Sound and Vibration, 322 (2009) 674-689.

[32] G. Squicciarini, M.G.R. Toward, D.J. Thompson, C.J.C. Jones, Statistical Description of Wheel Roughness, in: J. Nielsen, D. Anderson, P.-E. Gautier, M. Iida, J. Nelson, D. Thompson, T. Tielkes, D. Towers, P. de Vos (Eds.) Noise and Vibration Mitigation for Rail Transportation Systems, Springer Berlin Heidelberg, 2015, pp. 651-658. 